\title{
Pacific
}

Journal of

Mathematics

\section{ON THE STRUCTURE OF TENSOR PRODUCTS OF $\ell_{p}$-SPACES}

\author{
Alvaro ARIAS AND JEFFrey D. FARMER
}

Volume $175 \quad$ No. 1 


\title{
ON THE STRUCTURE OF TENSOR PRODUCTS OF $\ell_{p}$-SPACES
}

\author{
Alvaro Arias AND JefF D. FARMER
}

\begin{abstract}
We examine some structural properties of (injective and projective) tensor products of $\ell_{p}$-spaces (projections, complemented subspaces, reflexivity, isomorphisms, etc.). We combine these results with combinatorial arguments to address the question of primarity for these spaces and their duals.
\end{abstract}

\section{Introduction.}

A Banach space $X$ is prime if every infinite-dimensional complemented subspace contains a further subspace which is isomorphic to $X$. A Banach space $X$ is said to be primary if whenever $X=Y \oplus Z, X$ is isomorphic to either $Y$ or $Z$. The classical examples of prime spaces are the spaces $\ell_{p}, 1 \leq p \leq \infty$. Many spaces derived from the $\ell_{p}$-spaces in various ways are primary (see for example $[\mathbf{A E O}]$ and $[\mathbf{C L}])$.

The primarity of $B(H)$ was shown by Blower [B] in 1990, and Arias [A] has recently developed further techniques which are used to prove the primarity of $c_{1}$, the space of trace class operators (this was first shown by Arazy [Ar1, Ar2]). It has become clear that these techniques are not naturally confined to a Hilbert space context; in the present paper we wish to extend the results to a variety of tensor products and operator spaces of $\ell_{p}$-spaces (and in some cases $\mathcal{L}_{p}$-spaces). We also include some related results.

Some of the intermediate propositions (on factoring operators through the identity) may actually be true for a wider class of Banach spaces (those with unconditional bases which have nontrivial lower and upper estimates). In fact, the combinatorial aspects of the factorization can be applied quite generally, and may have other applications. The proofs of primarity, however, rely on Pełczyński's decomposition method which is not so readily extended. We have thus kept mainly to the case of injective and projective tensor products of $\ell_{p}$ spaces throughout. The results we obtain apply to the growing study of polynomials on Banach spaces since polynomials may be considered as symmetric multilinear operators with an equivalent norm (see $[\mathbf{F J}],[\mathbf{M}]$, or $[\mathbf{R}])$.

Our main results are:

(1) If $1<p<\infty$, then $B\left(\ell_{p}\right) \approx B\left(L_{p}\right)$. 
(2) If $\frac{1}{p_{i}}+\frac{1}{p_{j}} \leq 1$ for every $i \neq j$, or if all of the $p_{i}$ 's are equal, then $\ell_{p_{1}} \hat{\otimes} \cdots \hat{\otimes} \ell_{p_{N}}$ is primary.

(3) $\ell_{p}$ embeds into $\ell_{p_{1}} \hat{\otimes} \cdots \hat{\otimes} \ell_{p_{N}}$ if and only if there exists $A \subset\{1,2, \cdots, n\}$ such that $\frac{1}{p}=\min \left\{\sum_{i \in A} \frac{1}{p_{i}}, 1\right\}$.

(4) If $1 \leq p<\infty$ and $m \geq 1$, then the space of homogeneous analytic polynomials $\mathcal{P}_{m}\left(\ell_{p}\right)$ and the symmetric tensor product of $m$ copies of $\ell_{p}$ are primary.

The paper is organized as follows. In Section 1 we set notation, definitions and some necessary but more or less known facts. In Section 2 we show that $B\left(\ell_{p}\right)$, the Banach space of bounded linear operators on $\ell_{p}$, is isomorphic to $B\left(L_{p}\right)$, and in fact to $B(X)$ whenever $X$ is a separable $\mathcal{L}_{p}$-space, along with some more general results we require later. In Section 3 we will construct a multiplier through which a given operator on tensor products may be factored; we then use this to show that some projective tensor products are primary. In Section 4 we will prove that the $\ell_{p}$ subspaces of $\ell_{p_{1}} \hat{\otimes} \cdots \hat{\otimes} \ell_{p_{N}}$ are the "obvious" ones and use this to prove that some projective tensor products are not primary (for example, $\ell_{2} \hat{\otimes} \ell_{1.5}$ is not primary). Section 5 covers the question of primarity in the injective tensor products and operator spaces, a situation not always dual to the projective case and calling for somewhat different techniques. Section 6 is an appendix in which we prove the technical lemmas we use in Section 3.

We would like to thank W.B. Johnson for organizing the summer workshops in Linear Analysis and Probability at Texas A\&M University in 19911993, and the NSF for funding them.

\section{Preliminaries.}

Unless explicitly stated, all references to $\ell_{p}$ spaces will assume that $1<p<$ $\infty$, and will adhere the notational convention that $\frac{1}{p_{i}}+\frac{1}{q_{i}}=1$ or sometimes $\frac{1}{r}+\frac{1}{r^{\prime}}=1$.

Define

$$
X=\ell_{p_{1}} \hat{\otimes} \cdots \hat{\otimes} \ell_{p_{N}}
$$

We can identify its predual $X_{*}$ and dual $X^{*}$ as follows

$$
\begin{aligned}
X_{*} & =\ell_{q_{1}} \check{\otimes} \cdots \check{\otimes} \ell_{q_{N}} \\
X^{*} & =B\left(\ell_{p_{1}},\left(\ell_{p_{2}} \hat{\otimes} \cdots \hat{\otimes} \ell_{p_{N}}\right)^{*}\right) \\
& \equiv B\left(\ell_{p_{1}}, B\left(\cdots B\left(\ell_{p_{N-1}}, \ell_{q_{N}}\right) \cdots\right)\right) .
\end{aligned}
$$

The elements of $X, X_{*}$, or $X^{*}$ have representations as an infinite $N$-dimensional matrix of complex numbers (we must keep in mind, however, that this representation may not be the most efficient for computing the tensor 
product norm) where the element in the $\alpha=\left(\alpha_{1}, \cdots, \alpha_{N}\right) \in \mathbf{N}^{N}$ position is the coefficient of the "matrix element" $e_{\alpha}=e_{\alpha_{1}} \otimes \cdots \otimes e_{\alpha_{N}}$ with $e_{\alpha_{j}}$ being the $\alpha_{j}$-th element in the unit vector basis of $\ell_{p_{j}}$. All subspaces we consider are norm-closed, and when we indicate the linear span of elements we always mean the closed span.

The following elementary lemma is very important to the structure of projective tensor products.

Lemma 1.1. Let $X$ and $Y$ be Banach spaces and $S \in B(X), T \in B(Y)$. Then $S \otimes T \in B(X \hat{\otimes} Y)$ is defined by $S \otimes T(x \otimes y)=S(x) \otimes T(y)$ and satisfies $\|S \otimes T\| \leq\|S\|\|T\|$.

As a consequence of this we get that projective tensor products of Banach spaces with bases have bases.

Proposition 1.2. Let $X$ and $Y$ be Banach spaces with bases $\left(e_{n}\right)_{n}$ and $\left(f_{n}\right)_{n}$ respectively. Then $X \hat{\otimes} Y$ has a basis. Moreover, we take the elements of the basis from the "shell" $\partial M_{n}=\left[e_{i} \otimes e_{j}: \max \{i, j\}=n\right]$; i.e., $e_{1} \otimes f_{1}$, $e_{2} \otimes f_{1}, e_{2} \otimes f_{2}, e_{1} \otimes f_{2}, \quad e_{3} \otimes f_{1}, e_{3} \otimes f_{2}, e_{3} \otimes f_{3}, e_{2} \otimes f_{3}, e_{1} \otimes f_{3}, \cdots$, etc.

The proof of this is easy. On the one hand it is clear that the span of those vectors is dense and using Lemma 1.1 (with the operators replaced by projections) we see that the initial segments are uniformly complemented, because $\partial M_{n}$ is clearly complemented.

As a consequence we get that $\ell_{p_{1}} \hat{\otimes} \cdots \hat{\otimes} \ell_{p_{N}}$ has a basis consisting of $e_{\alpha}$ 's. Moreover, we can use Lemma 1.1 to prove that

$$
\partial M_{n}=\left[e_{\alpha}: \alpha \in \mathbf{N}^{N}, \max \left\{\alpha_{1}, \cdots, \alpha_{N}\right\}=n\right]
$$

is 2-complemented and that $\left(\partial M_{n}\right)_{n}$ forms a Schauder decomposition for $\ell_{p_{1}} \hat{\otimes} \cdots \hat{\otimes} \ell_{p_{N}}$; we also see that $\left(L_{\alpha}\right)_{\alpha}$ is a Schauder decomposition for $\ell_{p_{1}} \hat{\otimes} \cdots \hat{\otimes} \ell_{p_{N}}$ where $\alpha \in \mathbf{N}^{N-1}$ and $L_{\alpha}=\left[e_{\alpha} \otimes e_{j}: j \in \mathbf{N}\right]$. (A more complete discussion of this situation appears in $[\mathbf{R}]$.) We will use these facts in Section 3.

The next theorem gives us the two most basic ingredients of our analysis. We will prove that the main diagonals are 1-complemented and will identify them exactly; we will also state under what conditions the triangular parts of $\ell_{p_{1}} \hat{\otimes} \cdots \hat{\otimes} \ell_{p_{N}}$ are complemented. It is known that the main triangular part of $\ell_{p} \hat{\otimes} \ell_{q}$ is complemented if and only if $\frac{1}{p}+\frac{1}{q}>1$. (See [KP], [MN] and $[\mathbf{B e}]$.)

Theorem 1.3. Let $X=\ell_{p_{1}} \hat{\otimes} \cdots \hat{\otimes} \ell_{p_{N}}$. Then the main diagonal $\mathcal{D}=$ $\left[e_{n} \otimes \cdots \otimes e_{n}: n \in \mathbf{N}\right]$ is 1-complemented and satisfies $\mathcal{D} \equiv \ell_{r}$ where $\frac{1}{r}=$ 
$\min \left\{1, \sum_{i=1}^{N} \frac{1}{p_{i}}\right\}$. As a consequence we get that $X \approx\left(\sum \oplus X\right)_{r}$. Moreover, if $j, k$ are fixed, then the canonical projection onto

$$
\left[e_{i_{1}} \otimes e_{i_{2}} \otimes \cdots \otimes e_{i_{N}}: i_{k} \geq i_{j}\right]
$$

is bounded if and only if $\frac{1}{p_{k}}+\frac{1}{p_{j}}>1$.

This theorem is known for $n=2$, and in some respects for larger $n$ as well (see for example [Z]). For completeness we show here how the case $n=2$ may be extended.

Proof. For $1<k \leq N$, let $P_{1, k} \in B\left(\ell_{p_{1}} \hat{\otimes} \ell_{p_{k}}\right)$ be the main diagonal projection and $I_{1, k}$ be the identity on $\ell_{p_{2}} \hat{\otimes} \cdots \hat{\otimes} \ell_{p_{k-1}} \hat{\otimes} \ell_{p_{k+1}} \hat{\otimes} \cdots \hat{\otimes} \ell_{p_{N}}$. Then $P_{1, k} \otimes I_{1, k}$ is the projection on $\ell_{p_{1}} \hat{\otimes} \cdots \hat{\otimes} \ell_{p_{N}}$ defined by $P_{1, k} \otimes I_{1, k} e_{\alpha}=e_{\alpha}$ if $\alpha_{1}=\alpha_{k}$ and zero otherwise.

Let $P=\left(P_{1,2} \otimes I_{1,2}\right) \cdots\left(P_{1, N} \otimes I_{1, N}\right)$. It is easy to see that $P e_{\alpha}=e_{\alpha}$ if $\alpha_{1}=\cdots=\alpha_{N}$ and $P e_{\alpha}=0$ otherwise. This tells us that $\mathcal{D}$ is complemented.

When $N=2$, the main diagonal of $\ell_{p_{1}} \hat{\otimes} \ell_{p_{2}}$ is isometric to $\ell_{r}$ where $\frac{1}{r}=\min \left\{1, \frac{1}{p_{1}}+\frac{1}{p_{2}}\right\}$. We apply an induction step for $N>2$. The key to the induction step is the following: Let $D$ be the "diagonal-projection" on a projective tensor products of $\ell_{p}$-spaces. Then it is easy to see that $D\left(\ell_{p_{1}} \hat{\otimes} \cdots \hat{\otimes} \ell_{p_{N}}\right) \equiv D\left(D\left(\ell_{p_{1}} \hat{\otimes} \cdots \hat{\otimes} \ell_{p_{N-1}}\right) \hat{\otimes} \ell_{p_{N}}\right)$.

Notice that if the $P_{1, k}$ 's above are block projections, then we conclude that the block diagonal projections are bounded. By taking those to be infinite and using the previous paragraph, we see that $X \approx\left(\sum \oplus X\right)_{r}$.

For the last part let $T_{k, j}$ be the upper triangular projection on $\ell_{p_{k}} \hat{\otimes} \ell_{p_{j}}$ and $I_{k, j}$ be the identity on $\hat{\otimes}_{i \neq k, j} \ell_{p_{i}} . T_{k, j}$ is bounded if and only if $\frac{1}{p_{k}}+\frac{1}{p_{j}} \leq 1$. Therefore, the same is true for $T_{k, j} \otimes I_{k, j} \in B\left(\ell_{p_{1}} \hat{\otimes} \cdots \hat{\otimes} \ell_{p_{N}}\right)$.

Remarks. (1) To prove that $X \approx\left(\sum \oplus X\right)_{r}$ we used Pełczyński's decomposition method. This says that if two Banach spaces $X_{1}$ and $X_{2}$ embed complementably into each other and if for some $1 \leq p \leq \infty, X_{1} \approx\left(\sum \oplus X_{1}\right)_{p}$, then $X_{1} \approx X_{2}$.

(2) We will work in Section 3 with $\mathcal{T}=\left[e_{\alpha}: \alpha_{1}<\alpha_{2}<\cdots<\alpha_{N}\right]$. Some of the results from Theorem 1.3 hold for this space. For instance, the block projections are bounded. This implies that $\mathcal{T} \approx\left(\sum \mathcal{T}\right)_{r}$ where $r$ is as in Theorem 1.3.

(3) It is clear that when $r=1$ then $\ell_{p_{1}} \hat{\otimes} \cdots \hat{\otimes} \ell_{p_{N}}$ is not reflexive. It is not very difficult to prove that if $r>1$ then $\ell_{p_{1}} \hat{\otimes} \cdots \hat{\otimes} \ell_{p_{N}}$ is reflexive.

\section{Isomorphisms of Spaces of Operators on $\ell_{p}$.}

In this section we will show that $B\left(\ell_{p}\right)$ is isomorphic to $B(X)$ when $X$ is any separable $\mathcal{L}_{p}$-space. In particular, $B\left(\ell_{p}\right)$ is isomorphic to $B\left(L_{p}[0,1]\right)$. 
A consequence of this is that $B\left(\ell_{2}\right)$ embeds complementably in $B\left(\ell_{p}\right)$ for $1<p<\infty$.

Theorem 2.1. Let $X$ and $Y$ be separable $\mathcal{L}_{p}$-and $\mathcal{L}_{q}$-spaces respectively with $1<p \leq q$. Then $B(X, Y) \approx B\left(\ell_{p}, \ell_{q}\right) \approx\left(\sum_{n=1}^{\infty} \oplus B\left(\ell_{p}^{n}, \ell_{q}^{n}\right)\right)_{\infty}$.

We also obtain an isomorphic representation for $\left(\ell_{p_{1}} \hat{\otimes} \cdots \hat{\otimes} \ell_{p_{N}}\right)^{*}$ when $\sum_{i \leq N} \frac{1}{p_{i}} \geq 1$.

Theorem 2.2. Let $X=\ell_{p_{1}} \hat{\otimes} \cdots \hat{\otimes} \ell_{p_{N}}$ be such that $\frac{1}{r}=\min \left\{1, \sum_{i=1}^{N} \frac{1}{p_{i}}\right\}=$ 1. Then $X^{*} \approx\left(\sum_{n=1}^{\infty} \ell_{q_{1}}^{n} \check{\otimes} \cdots \check{\otimes} \ell_{q_{N}}^{n}\right)_{\infty}$.

The proof of these two theorems is very similar; they use Petczyński's decomposition method.

For Theorem 2.1 notice that $B\left(\ell_{p}, \ell_{q}\right) \equiv\left(\ell_{p} \hat{\otimes} \ell_{q^{\prime}}\right)^{*}$ where $\frac{1}{q}+\frac{1}{q^{\prime}}=1$. Hence, if $p \leq q$ (i.e., $\frac{1}{p}+\frac{1}{q^{\prime}} \geq 1$ ), Theorem 1.3 tells us that $\ell_{p} \hat{\otimes} \ell_{q^{\prime}} \approx\left(\sum \oplus \ell_{p} \hat{\otimes} \ell_{q^{\prime}}\right)_{1}$, and then $B\left(\ell_{p}, \ell_{q}\right) \approx\left(\sum \oplus B\left(\ell_{p}, \ell_{q}\right)\right)_{\infty}$. For Theorem 2.2, notice that Theorem 1.3 implies that $X \approx\left(\sum \oplus X\right)_{1}$; therefore, $X^{*} \approx\left(\sum \oplus X^{*}\right)_{\infty}$.

Then it is enough to prove that each space embeds complementably into the other. We prove these facts for Theorem 2.1 in the next two lemmas and indicate how to do it for Theorem 2.2 at the end of the section.

A Banach space $X$ is $\mathcal{L}_{p}$ if its finite dimensional subspaces are like those of $\ell_{p}$. If $1<p<\infty$, the separable $\mathcal{L}_{p}$-spaces are the complemented subspaces of $L_{p}[0,1]$ not isomorphic to $\ell_{2}$.

We use the following properties of a separable $\mathcal{L}_{p}$-space $X:(1) X$ contains a complemented copy of $\ell_{p}$, and (2) There is an increasing (by inclusion) sequence of finite dimensional subspaces which are uniformly isomorphic to finite dimensional $\ell_{p}$-spaces. Moreover, they are uniformly complemented and their union is dense in $X$. For more information on $\mathcal{L}_{p}$-spaces see [LP] or [JRZ].

Lemma 2.3. Suppose that $1<p \leq q$ and let $X$ and $Y$ be separable $\mathcal{L}_{p}$ or $\mathcal{L}_{q}$ spaces. Then $B(X, Y)$ embeds complementably in $W=\left(\sum_{n=1}^{\infty} \oplus B\left(\ell_{p}^{n}, \ell_{q}^{n}\right)\right)_{\infty}$.

Proof. By the assumptions on $X$ and $Y$, we can find $\phi_{n}: B\left(\ell_{p}^{n}, \ell_{q}^{n}\right) \rightarrow B(X, Y)$ and $\psi_{n}: B(X, Y) \rightarrow B\left(\ell_{p}^{n}, \ell_{q}^{n}\right)$ satisfying: (1) $\psi_{n} \phi_{n}=I_{n}$, the identity on $B\left(\ell_{p}^{n}, \ell_{q}^{n}\right)$, and (2) for every $T \in B(X, Y), \phi_{n} \psi_{n}(T) \rightarrow T$ in the $w^{*}$-topology.

Then define $\Psi: B(X, Y) \rightarrow W$ by $\Psi(T)=\left(\psi_{n}(T)\right)_{n}$. Let $\mathcal{U}$ be a free ultrafilter in $\mathbf{N}$ and define $\Phi: W \rightarrow B(X, Y)$ by $\Phi\left(\left(T_{n}\right)\right)=\lim _{n \in \mathcal{U}} \phi_{n}\left(T_{n}\right)$ where the limit is taken in the $w^{*}$-topology. We can easily verify that $\Phi \Psi=$ $I$, the identity on $B(X, Y)$, and the conclusion follows. 
Lemma 2.4. Let $X$ and $Y$ be $\mathcal{L}_{p}$ and $\mathcal{L}_{q}$-spaces respectively, with $1<p \leq q$ and let $W$ be as above. Then $W$ embeds complementably into $B(X, Y)$

Proof. It is clear that $W$ embeds complementably into $B\left(\ell_{p}, \ell_{q}\right)$, because $B\left(\ell_{p}, \ell_{q}\right)$ has $\ell_{\infty}$-blocks down the diagonal. Moreover, if $X$ is a separable $\mathcal{L}_{p}$-space, $1<p<\infty$, then $X$ contains a complemented copy of $\ell_{p}$. Since the same is true for $Y$ we see that $B\left(\ell_{p}, \ell_{q}\right)$ embeds complementably into $B(X, Y)$.

Remark. For Theorem 2.2 notice that

$$
Z_{n}=B\left(\ell_{p_{1}}^{n}, B\left(\cdots B\left(\ell_{p_{N-1}}^{n}, \ell_{q_{N}}^{n}\right) \cdots\right)\right)
$$

is isometric to $\ell_{q_{1}}^{n} \check{\otimes} \cdots \check{\otimes} \ell_{q_{N}}^{n}$ and is 1-complemented in $X^{*}$. (We use this to show that $\left(\sum_{n} \oplus Z_{n}\right)_{\infty}$ embeds complementably into $X^{*}$.) Moreover, $\bigcup_{n} Z_{n}$ is $w^{*}$-dense in $X^{*}$. (We may use this and an ultrafilter argument to show the reverse complemented inclusion.)

\section{Primarity of Projective Tensor Products.}

We devote most of this section to the proof of the following theorem.

Theorem 3.1. Let $X=\ell_{p_{1}} \hat{\otimes} \cdots \hat{\otimes} \ell_{p_{N}}$ be such that $\frac{1}{p_{i}}+\frac{1}{p_{j}} \leq 1$ for every $i \neq j$. Then $X$ is primary.

The proof of this theorem will follow easily from the next proposition that was inspired by results of Blower $[\mathbf{B}]$ and was used in $[\mathbf{A}]$ in a similar context. The ideas involved in this "factorization" approach are well-known (see for example Bourgain [Bo]).

We have to introduce some notation.

Let $X=\ell_{p_{1}} \hat{\otimes} \cdots \hat{\otimes} \ell_{p_{N}}, \alpha=\left(\alpha_{1}, \cdots, \alpha_{N}\right) \in \mathbf{N}^{N}$ and denote by $e_{\alpha}=$ $e_{\alpha_{1}} \otimes e_{\alpha_{2}} \otimes \cdots \otimes e_{\alpha_{N}}$. Then $X=\left[e_{\alpha}: \alpha \in \mathbf{N}^{N}\right]$. We also define $|\alpha|=$ $\max \left\{\alpha_{1}, \cdots \alpha_{2}\right\}$; and introduce an order between different multiindices. Let $\alpha \in \mathbf{N}^{k}$ and $\beta \in \mathbf{N}^{m}$; we say that

$$
\alpha<\beta \quad \text { if } \max \left\{\alpha_{1}, \cdots, \alpha_{k}\right\}<\min \left\{\beta_{1}, \cdots, \beta_{m}\right\} \text {. }
$$

Let $\sigma_{1}, \sigma_{2}, \cdots \sigma_{N}: \mathbf{N} \rightarrow \mathbf{N}$ be increasing functions (it will also be useful to think of the $\sigma_{i}$ 's as infinite subsets of $\left.\mathbf{N}\right)$; and let $\sigma=\left(\sigma_{1}, \sigma_{2}, \cdots, \sigma_{N}\right)$ be a function on $\mathbf{N}^{N}$ defined by $\sigma(\alpha)=\left(\sigma_{1}\left(\alpha_{1}\right), \cdots, \sigma_{N}\left(\alpha_{N}\right)\right)$. Then define

$$
\begin{aligned}
J_{\sigma}: \ell_{p_{1}} \hat{\otimes} \cdots \hat{\otimes} \ell_{p_{N}} & \rightarrow \ell_{p_{1}} \hat{\otimes} \cdots \hat{\otimes} \ell_{p_{N}} \\
K_{\sigma}: \ell_{p_{1}} \hat{\otimes} \cdots \hat{\otimes} \ell_{p_{N}} & \rightarrow \ell_{p_{1}} \hat{\otimes} \cdots \hat{\otimes} \ell_{p_{N}}
\end{aligned}
$$


by

$J_{\sigma} e_{\alpha}=e_{\sigma(\alpha)} \quad$ and $\quad K_{\sigma} e_{\alpha}= \begin{cases}e_{\beta} & \text { if there exists } \beta \text { such that } \sigma(\beta)=\alpha \\ 0 & \text { otherwise. }\end{cases}$

$J_{\sigma}$ and $K_{\sigma}$ have many important algebraic properties. $J_{\sigma}$ is one-to one, $K_{\sigma}$ is onto and $K_{\sigma} J_{\sigma}=I$. Moreover, they compose nicely; that is, if $\sigma=$ $\left(\sigma_{1}, \sigma_{2}, \cdots, \sigma_{N}\right)$ and $\psi=\left(\psi_{1}, \cdots, \psi_{N}\right)$, then

$$
J_{\sigma} J_{\psi}=J_{\sigma \psi} \quad \text { and } \quad K_{\sigma} K_{\psi}=K_{\sigma \psi} .
$$

We are now ready to state the proposition.

Proposition 3.2. Let $\frac{1}{p_{i}}+\frac{1}{p_{j}} \leq 1$ for every $i \neq j$. Then if $\Phi \in$ $B\left(\ell_{p_{1}} \hat{\otimes} \cdots \hat{\otimes} \ell_{p_{N}}\right)$ and $\epsilon>0$ there exist $\sigma=\left(\sigma_{1}, \sigma_{2}, \cdots, \sigma_{N}\right)$ and $\lambda \in \mathbf{C}$ such that

$$
\left\|K_{\sigma} \Phi J_{\sigma}-\lambda I\right\|<\epsilon .
$$

Thus one of $K_{\sigma} \Phi J_{\sigma}$ or $K_{\sigma}(\Phi-I) J_{\sigma}$ is invertible.

It is immediate from this proposition that $I$, the identity on $\ell_{p_{1}} \hat{\otimes} \cdots \hat{\otimes} \ell_{p_{N}}$, factors through $\Phi$ or through $I-\Phi$ which implies trivially that if $\ell_{p_{1}} \hat{\otimes} \cdots \hat{\otimes} \ell_{p_{N}} \approx X \oplus Y$ then $\ell_{p_{1}} \hat{\otimes} \cdots \hat{\otimes} \ell_{p_{N}}$ embeds complementably into $X$ or $Y$. Since $\ell_{p_{1}} \hat{\otimes} \cdots \hat{\otimes} \ell_{p_{N}}$ is isomorphic to its infinite $r$-sum, the Pełczyński decomposition method implies that $\ell_{p_{1}} \hat{\otimes} \cdots \hat{\otimes} \ell_{p_{N}}$ is primary.

We will present a sketch of the proof. For $\Phi \in \ell_{p_{1}} \hat{\otimes} \cdots \hat{\otimes} \ell_{p_{N}}$ and $\alpha \in \mathbf{N}^{N}$ we have,

$$
\Phi e_{\alpha}=\sum_{\beta \in \mathbf{N}^{N}} \lambda_{\alpha, \beta} e_{\beta},
$$

for some $\lambda_{\alpha, \beta} \in \mathbf{C}$. Our goal will be to come with a series of the aforementioned $J$-maps and $K$-maps which will allow us to get $K \Phi J \approx \lambda I$. We will do this is several steps, fixing progressively more restrictive portions of the range of $\beta$. We can do this since this maps compose nicely; however we must be careful not to destroy previous work (see the assumption below). More precisely, Step 1 asserts that we can find $K_{1}, J_{1}$ such that $K_{1} \Phi J_{1} \approx \Phi_{1}$ and for every $n \in \mathbf{N}$,

$$
\alpha \in \mathbf{N}^{N}, \quad|\alpha|=n \quad \Longrightarrow \quad \Phi_{1} e_{\alpha}=\sum_{|\beta|=n} \lambda_{\alpha, \beta}^{(1)} e_{\beta},
$$

for some $\lambda_{\alpha, \beta}^{(1)} \in \mathbf{C}$. This is clearly an improvement in the range of $\beta$, but we still have that $\left\{\beta \in \mathbf{N}^{N}:|\beta|=n\right\}$ is a big set. After Steps 2,3 and 4 we 
have $K_{4}, J_{4}$ such that $K_{4} \Phi J_{4} \approx \Phi_{4}$ and for every $n \in \mathbf{N}, j \in \mathbf{N}$,

$$
\alpha \in \mathbf{N}^{N-1}, \quad|\alpha|=n, \quad|\alpha|<j \Longrightarrow \Phi_{4}\left(e_{\alpha} \otimes e_{j}\right)=\sum_{\substack{|\beta|=n \\ \beta \in N^{N-1}}} \lambda_{\alpha, \beta}^{(4)} e_{\beta} \otimes e_{j} .
$$

Step 5 gives us $K_{5}, J_{5}$ such that $K_{5} \Phi J_{5} \approx \Phi_{5}$ and for every $n \in \mathbf{N}, \gamma \in \mathbf{N}^{2}$,

$$
\alpha \in \mathbf{N}^{N-2}, \quad|\alpha|=n, \quad \alpha<\gamma \Longrightarrow \Phi_{5}\left(e_{\alpha} \otimes e_{\gamma}\right)=\sum_{\substack{|\beta|=n \\ \beta \in \mathbb{N}^{N-2}}} \lambda_{\alpha, \beta}^{(5)} e_{\beta} \otimes e_{\gamma} .
$$

Finally Step 6 provides the general induction argument.

We will apply our arguments on $\mathcal{T}=\left[e_{\alpha}: \alpha_{1}<\alpha_{2}<\cdots<\alpha_{N}\right]$ without loss of generality in order to simplify notation, keeping in mind that they will be repeated many times when the order of the $\alpha_{i}$ 's is different. We will choose $\sigma$ so that $J_{\sigma}$ and $K_{\sigma}$ "respect" that order. More precisely, consider the permutation group $\Pi_{n}$ and a multiindex $\alpha=\left(\alpha_{1}, \cdots, \alpha_{N}\right) \in$ $\mathbf{N}^{N}$. We choose $\sigma$ so that the (not necessarily complemented) subspaces $\mathcal{T}(\pi)=\left[e_{\alpha}: \alpha_{\pi(1)}<\alpha_{\pi(2)}<\cdots<\alpha_{\pi(N)}\right]$ are invariant for $J_{\sigma}$ and $K_{\sigma}$; i.e., $J_{\sigma} \mathcal{T}(\pi) \subset \mathcal{T}(\pi)$ and $K_{\sigma} \mathcal{T}(\pi) \subset \mathcal{T}(\pi)$. Notice that the $\mathcal{T}(\pi)$ 's "exhaust" the $N$-dimensional matrix array on which we represent $\ell_{p_{1}} \hat{\otimes} \cdots \hat{\otimes} \ell_{p_{N}}$ (modulo diagonal elements, which we always ignore; see Step 2).

Assumption. Assume from now on that whenever we choose

$$
\sigma=\left(\sigma_{1}, \sigma_{2}, \cdots, \sigma_{N}\right),
$$

it always "preserves the order", that is, if $i<j$, then $\sigma_{k}(i)<\sigma_{l}(j)$, for every $k, l \leq N$.

We can always satisfy this assumption by passing to subsequences whenever we are choosing the sets $\sigma$, which our technical lemmas allow us to do.

Example. It might be instructive to consider the following example "far" from a multiplier. Let $\Phi: \ell_{2} \hat{\otimes} \ell_{2} \rightarrow \ell_{2} \hat{\otimes} \ell_{2}$ be the transpose operator, i.e., $\Phi e_{i} \otimes e_{j}=e_{j} \otimes e_{i}$. Then choose $\sigma_{1}$ the set of even integers, $\sigma_{2}$ the set of odd integers and $\sigma=\left(\sigma_{1}, \sigma_{2}\right)$. We verify easily that $K_{\sigma} \Phi J_{\sigma}=0$ thus satisfying the conclusion of Proposition 3.2.

Our steps require the repeated use of two technical lemmas whose proof we delay until Section 6 .

Step 1. Let $\partial M_{n}=\left[e_{\alpha}: \alpha \in \mathbf{N}^{N},|\alpha|=n\right]$ with projection $Q_{n}$. Then for every $\Phi \in B\left(\ell_{p_{1}} \hat{\otimes} \cdots \hat{\otimes} \ell_{p_{N}}\right)$ and $\epsilon>0$, there exist $\sigma=\left(\sigma_{1}, \sigma_{2}, \cdots, \sigma_{N}\right)$ and $\Phi_{1} \in B\left(\ell_{p_{1}} \hat{\otimes} \cdots \hat{\otimes} \ell_{p_{N}}\right)$ such that $\left\|\Phi_{1}-K_{\sigma} \Phi J_{\sigma}\right\|<\epsilon$ and for every $n$, $\Phi_{1} \partial M_{n} \subset \partial M_{n}$. 
The proof of this step is an immediate consequence of the following lemma. Remember that $\left(\partial M_{n}\right)_{n}$ forms a Schauder decomposition for $\ell_{p_{1}} \hat{\otimes} \cdots \hat{\otimes} \ell_{p_{N}}$.

Basic Lemma 1. Let $\Phi \in B\left(\ell_{p_{1}} \hat{\otimes} \cdots \hat{\otimes} \ell_{p_{N}}\right)$. Then for every $\epsilon_{n, m}>0$ we can find $\sigma=\left(\sigma_{1}, \sigma_{2}, \cdots, \sigma_{N}\right)$ such that if $x \in \partial M_{n}$, and $n \neq m$, then $\left\|Q_{m} K_{\sigma} \Phi J_{\sigma} x\right\| \leq \epsilon_{n, m}\|x\|$.

We prove Basic Lemma 1 in the appendix (if $X=\ell_{p}$ the proof is very easy).

Choose $\epsilon_{n, m}$ in Basic Lemma 1 so that $\epsilon_{n}=\sum_{m=1}^{\infty} \epsilon_{n, m}$ and $\sum_{n=1}^{\infty} \epsilon_{n}<\frac{\epsilon}{2}$. Then define $\Phi_{1}$ on $\ell_{p_{1}} \hat{\otimes} \cdots \hat{\otimes} \ell_{p_{N}}$ as follows: For $x \in \partial M_{n}$, let

$$
\Phi_{1}(x)=Q_{n} K_{\sigma} \Phi J_{\sigma} x .
$$

If $x \in \partial M_{n}$, then $\left\|\left(\Phi_{1}-K_{\sigma} \Phi J_{\sigma}\right) x\right\| \leq \epsilon_{n}\|x\|$. If $x \in \ell_{p_{1}} \hat{\otimes} \cdots \hat{\otimes} \ell_{p_{N}}$, we have that $x=\sum_{n=1}^{\infty} x_{n}$ where $x_{n} \in \partial M_{n}$ and $\left\|x_{n}\right\| \leq 2\|x\|$. Therefore,

$$
\left\|\left(\Phi_{1}-K_{\sigma} \Phi J_{\sigma}\right) x\right\| \leq \sum_{n=1}^{\infty}\left\|\left(\Phi_{1}-K_{\sigma} \Phi J_{\sigma}\right) x_{n}\right\|<\epsilon\|x\| .
$$

Step 2. Let $\Phi \in B\left(\ell_{p_{1}} \hat{\otimes} \cdots \hat{\otimes} \ell_{p_{N}}\right)$ be such that $\Phi \partial M_{n} \subset \partial M_{n}$ for every $n$, then we can find $\sigma=\left(\sigma_{1}, \sigma_{2}, \cdots, \sigma_{N}\right)$ such that $\Phi_{2}=K_{\sigma} \Phi J_{\sigma}$ "respects" the place where $\alpha \in \mathbf{N}^{N}$ takes its maximum; for example, if the maximum takes place in the last coordinate, i.e., $\alpha \in \mathbf{N}^{N-1}$ and $|\alpha|<j$, then

$$
\Phi_{2}\left(e_{\alpha} \otimes e_{\jmath}\right)=\sum_{|\beta|<j} \lambda_{\alpha, \beta, j} e_{\beta} \otimes e_{j},
$$

and we also have similar results for the other coordinates.

We attain this by "disjointifying" the different faces. For $i \leq N$ let $\sigma_{i}(j)=$ $N(j-1)+i$, and $\sigma=\left(\sigma_{1}, \sigma_{2}, \cdots, \sigma_{N}\right)$. It is easy to see that $\Phi_{2}=K_{\sigma} \Phi J_{\sigma}$ satisfies the required property. Indeed, if $\alpha \in \mathbf{N}^{N-1}$ and $|\alpha|<n$, then $e_{\alpha} \otimes e_{n} \in \partial M_{n}$, and $J_{\sigma}\left(e_{\alpha} \otimes e_{n}\right) \in \partial M_{\sigma_{N}(n)}$. Hence,

$$
\Phi J_{\sigma}\left(e_{\alpha} \otimes e_{n}\right)=\sum_{|\gamma|=\sigma_{N}(n)} \lambda_{\sigma(\alpha, n), \gamma} e_{\gamma} .
$$

Recall that $K_{\sigma} e_{\gamma}=e_{\eta}$ if $\sigma(\eta)=\gamma$ for some $\eta$ and $K_{\sigma} e_{\gamma}=0$ otherwise. Since the ranges of the $\sigma_{i}$ 's are disjoint, $\sigma_{N}(n)$ is nonzero only for the last coordinate. Therefore, if $|\gamma|=\sigma_{N}(n)$ and $\sigma(\eta)=\gamma$, the last coordinate of $r$ must be $n$; i.e., $e_{\eta}=e_{\beta} \otimes e_{n}$ for some $\beta \in \mathbf{N}^{N-1}$, and since $\sigma$ preserves the order, $|\beta|<n$. That is,

$$
K_{\sigma} \Phi J_{\sigma}\left(e_{\alpha} \otimes e_{n}\right)=\sum_{|\beta|<n} \lambda_{\sigma(\alpha, n), \sigma(\beta, n)} e_{\beta} \otimes e_{n} .
$$


We denote $\lambda_{\sigma(\alpha, n), \sigma(\beta, n)}$ by $\lambda_{\alpha, \beta, n}$.

To make the notation a bit clearer we will state the hypothesis and the conclusion of the steps when the maximum takes place in the $N$ th coordinate. However the other cases are identical and we will assume that (after repeating the step for the other coordinates) the same result holds for these cases.

Step 3. Let $\Phi \in B\left(\ell_{p_{1}} \hat{\otimes} \cdots \hat{\otimes} \ell_{p_{N}}\right)$ be such that whenever $\alpha \in \mathbf{N}^{N-1}, j \in \mathbf{N}$ satisfy $|\alpha|<j$, then $\Phi\left(e_{\alpha} \otimes e_{j}\right)=\sum_{|\beta|<j} \lambda_{\alpha, \beta, j} e_{\beta} \otimes e_{j}$. Then for every $\epsilon>0$ there exist $\sigma=\left(\sigma_{1}, \sigma_{2}, \cdots, \sigma_{N}\right)$ and $\Phi_{3} \in B\left(\ell_{p_{1}} \hat{\otimes} \cdots \hat{\otimes} \ell_{p_{N}}\right)$ such that $\left\|\Phi_{3}-K_{\sigma} \Phi J_{\sigma}\right\|<\epsilon$ and whenever $\alpha \in \mathbf{N}^{N-1}, j \in \mathbf{N}$ satisfy $|\alpha|<j$, then

$$
\Phi_{3}\left(e_{\alpha} \otimes j\right)=\sum_{|\beta|<j} \mu_{\alpha, \beta} e_{\beta} \otimes j .
$$

The proof of this step follows from the next lemma.

Basic Lemma 2. Let $\Phi \in B\left(\ell_{p_{1}} \hat{\otimes} \cdots \hat{\otimes} \ell_{p_{N}}\right)$ be such that whenever $\alpha \in$ $\mathbf{N}^{N-1}, j \in \mathbf{N}$ satisfy $|\alpha|<j$, then $\Phi\left(e_{\alpha} \otimes e_{j}\right)=\sum_{|\beta|<j} \lambda_{\alpha, \beta, j} e_{\beta} \otimes e_{j}$. Then for every $\epsilon_{\alpha, \beta, j}>0$ with $j>\max \{|\alpha|,|\beta|\}$, we can find $\sigma=\left(\sigma_{1}, \sigma_{2}, \cdots, \sigma_{N}\right)$ (respecting the order) such that if we set $\tilde{\sigma}=\left(\sigma_{1}, \sigma_{2}, \cdots, \sigma_{N-1}\right)$ then

$$
\begin{gathered}
\lim _{j \rightarrow \infty} \lambda_{\tilde{\sigma}(\alpha), \tilde{\sigma}(\beta), \sigma_{N}(j)}=\lambda_{\tilde{\sigma}(\alpha), \tilde{\sigma}(\beta)} \\
\left|\lambda_{\tilde{\sigma}(\alpha), \tilde{\sigma}(\beta), \tilde{\sigma}_{N}(j)}-\lambda_{\tilde{\sigma}(\alpha), \tilde{\sigma}(\beta)}\right| \leq \epsilon_{\alpha, \beta, j} .
\end{gathered}
$$

We also give the proof of Basic Lemma 2 in the appendix. Then set $\tilde{\Phi}=K_{\sigma} \Phi J_{\sigma}$, and let $L_{\alpha}=\left[e_{\alpha} \otimes e_{j}: j \in \mathbf{N}\right]$ with projection $P_{\alpha}$. Since $\ell_{p_{1}} \hat{\otimes} \cdots \hat{\otimes} \ell_{p_{N-1}}$ has a basis consisting of $e_{\alpha}$ 's, we have that $\left(L_{\alpha}\right)_{\alpha}$ forms a Schauder decomposition for $\ell_{p_{1}} \hat{\otimes} \cdots \hat{\otimes} \ell_{p_{N}}$.

Define $\Phi_{3} \in B\left(\ell_{p_{1}} \hat{\otimes} \cdots \hat{\otimes} \ell_{p_{N}}\right)$ by

$$
P_{\beta} \Phi_{3}\left(e_{\alpha} \otimes e_{j}\right)= \begin{cases}\lambda_{\tilde{\sigma}(\alpha), \tilde{\sigma}(\beta)} e_{\beta} \otimes e_{j} & \text { if } \max \{|\alpha|,|\beta|\}<j ; \\ P_{\beta} \tilde{\Phi}\left(e_{\alpha} \otimes e_{j}\right) & \text { otherwise. }\end{cases}
$$

Let $\alpha, \beta \in \mathbf{N}^{N} ; \epsilon_{\alpha, \beta}=\sum_{j>\max \{|\alpha|,|\beta|\}} \epsilon_{\alpha, \beta, j} ;$ and $x \in L_{\alpha} ;$ i.e., $x=\sum_{j=1}^{\infty} e_{\alpha} \dot{\otimes}$ $c_{j} e_{j}$. Then,

$$
P_{\beta} \Phi_{3} x-P_{\beta} \tilde{\Phi} x=\sum_{j>\max \{|\alpha|,|\beta|\}} e_{\beta} \otimes\left(\lambda_{\tilde{\sigma}(\alpha), \tilde{\sigma}(\beta)}-\lambda_{\tilde{\sigma}(\alpha), \tilde{\sigma}(\beta), \sigma_{N}(j)}\right) c_{j} e_{j}
$$


Hence,

$$
\left\|P_{\beta} \Phi_{3} x-P_{\beta} \tilde{\Phi} x\right\| \leq \sum_{j>\max \{|\alpha|,|\beta|\}} \epsilon_{\alpha, \beta, j} \max \left|c_{j}\right| \leq \epsilon_{\alpha, \beta}\|x\| .
$$

If we choose $\sum_{\alpha} \sum_{\beta} \epsilon_{\alpha, \beta}<\epsilon$ small enough, $\Phi_{3}$ is well defined and satisfies the required properties.

Step 4. Let $\Phi \in B\left(\ell_{p_{1}} \hat{\otimes} \cdots \hat{\otimes} \ell_{p_{N}}\right)$ be such that whenever $\alpha \in \mathbf{N}^{N-1}$, $j \in \mathbf{N}$ satisfy $|\alpha|<j$, then $\Phi\left(e_{\alpha} \otimes e_{j}\right)=\sum_{|\beta|<j} \lambda_{\alpha, \beta} e_{\beta} \otimes e_{j}$. Then for every $\epsilon>0$, there exist $\sigma=\left(\sigma_{1}, \sigma_{2}, \cdots, \sigma_{N}\right)$ and $\Phi_{4} \in B\left(\ell_{p_{1}} \hat{\otimes} \cdots \hat{\otimes} \ell_{p_{N}}\right)$ such that $\left\|\Phi_{4}-K_{\sigma} \Phi J_{\sigma}\right\|<\epsilon$ and whenever $\alpha \in \mathbf{N}^{N-1}, j \in \mathbf{N}$ satisfy $|\alpha|<j$, we have

$$
\Phi_{4}\left(e_{\alpha} \otimes e_{j}\right)=\sum_{|\beta|=|\alpha|} \mu_{\alpha, \beta} e_{\beta} \otimes e_{j} .
$$

Define $\Psi \in B\left(\ell_{p_{1}} \hat{\otimes} \cdots \hat{\otimes} \ell_{p_{N-1}}\right)$ by

$$
\left(\Psi e_{\alpha}, e_{\beta}\right)=\lambda_{\alpha, \beta}
$$

Since $\left(\Psi e_{\alpha}, e_{\beta}\right)=\lim _{j \rightarrow \infty}\left(\Phi e_{\alpha} \otimes e_{j}, e_{\beta} \otimes e_{j}\right), \Psi$ is a bounded map.

Remark. Ideally we would like to apply an induction step and replace $\Psi$, after a factorization of the form $K \Psi J$, by a multiple of the identity and then combine this with $\Phi$. Controlling the norm of the perturbation requires a more delicate argument, however.

Apply Basic Lemma 1 to $\Psi$ with its respective $\partial M_{n}$ and projections $Q_{n}$; then find $\sigma=\left(\sigma_{1}, \sigma_{2}, \cdots, \sigma_{N-1}\right)$ such that whenever $x \in \partial M_{n}$, and $m \neq n$,

$$
\left\|Q_{m} K_{\sigma} \Psi J_{\sigma} x\right\| \leq \epsilon_{n, m}\|x\| \text {. }
$$

Let $\tilde{\Psi}=K_{\sigma} \Psi J_{\sigma}, \tilde{\sigma}=\left(\sigma_{1}, \cdots, \sigma_{N-1}, \sigma_{N-1}\right)$ and $\tilde{\Phi}=K_{\tilde{\sigma}} \Phi J_{\tilde{\sigma}}$. Denote by $P_{n}=Q_{1}+\cdots+Q_{n}$ the projection onto $\left[e_{\alpha}: \alpha \in \mathbf{N}^{N-1},|\alpha| \leq n\right]$. Notice now that if $|\alpha|<j$ then $\tilde{\Phi}\left(e_{\alpha} \otimes e_{j}\right)=\left(P_{j} \tilde{\Psi} e_{\alpha}\right) \otimes e_{j}$.

Let $L_{\alpha}=\left[e_{\alpha} \otimes e_{j}: j \in \mathbf{N}\right]$. Then as we explained after the Basic Lemma $2,\left(L_{\alpha}\right)_{\alpha}$ forms a Schauder decomposition for $\ell_{p_{1}} \hat{\otimes} \cdots \hat{\otimes} \ell_{p_{N}}$.

Define $\Phi_{4}$ on $\ell_{p_{1}} \hat{\otimes} \cdots \hat{\otimes} \ell_{p_{N}}$ as follows

$$
\Phi_{4}\left(e_{\alpha} \otimes e_{j}\right)= \begin{cases}\tilde{\Phi}\left(e_{\alpha} \otimes e_{j}\right) & \text { if }|\alpha| \geq j \\ \left(Q_{|\alpha|} \tilde{\Psi} e_{\alpha}\right) \otimes e_{j} & \text { if }|\alpha|<j\end{cases}
$$

Let $n \in \mathbf{N} ; \alpha \in \mathbf{N}^{N-1}$ with $|\alpha|=n ; \epsilon_{n}=\sum_{m=1, m \neq n}^{\infty} \epsilon_{n, m}$ and $x \in L_{\alpha}$; i.e., $x=\sum_{j=1}^{\infty} e_{\alpha} \otimes c_{j} e_{j}$. Then,

$$
\tilde{\Phi}(x)-\Phi_{4}(x)=\sum_{j>n}\left(P_{j} \tilde{\Psi} e_{\alpha}-Q_{n} \tilde{\Psi} e_{\alpha}\right) \otimes c_{j} e_{j}
$$




$$
\begin{aligned}
& =\sum_{j>n} \sum_{\substack{k=1 \\
k \neq n}}^{j}\left(Q_{k} \tilde{\Psi} e_{\alpha}\right) \otimes c_{j} e_{j} \\
& =\sum_{\substack{k=1 \\
k \neq n}}^{\infty}\left(Q_{k} \tilde{\Psi} e_{\alpha}\right) \otimes \sum_{j>\max \{k, n\}} c_{j} e_{j} .
\end{aligned}
$$

Since $\left\|\sum_{j>\max \{k, n\}} c_{j} e_{j}\right\| \leq\|x\|$, we have that

$$
\left\|\Phi_{4}(x)-\tilde{\Phi}(x)\right\| \leq \epsilon_{n}\|x\| .
$$

Since $\operatorname{card}\{\alpha:|\alpha|=n\}$ is finite, it is enough to choose $\epsilon_{n}$ so that

$$
\sum_{n=1}^{\infty} \operatorname{card}\{\alpha:|\alpha|=n\} \epsilon_{n}<\epsilon
$$

to insure that $\Phi_{4}$ is well defined and satisfies the required properties.

Step 5. Let $\Phi \in B\left(\ell_{p_{1}} \hat{\otimes} \cdots \hat{\otimes} \ell_{p_{N}}\right)$ be such that whenever $\alpha \in \mathbf{N}^{N-1}$ and $j \in \mathbf{N}$ satisfy $|\alpha|<j$, then $\Phi\left(e_{\alpha} \otimes e_{j}\right)=\sum_{|\beta|=|\alpha|} \lambda_{\alpha, \beta} e_{\beta} \otimes e_{j}$. Then for every $\epsilon>0$, there exist $\sigma=\left(\sigma_{1}, \sigma_{2}, \cdots, \sigma_{N}\right)$ and $\Phi_{5} \in B\left(\ell_{p_{1}} \hat{\otimes} \cdots \hat{\otimes} \ell_{p_{N}}\right)$ such that $\left\|\Phi_{5}-K_{\sigma} \Phi J_{\sigma}\right\|<\epsilon$ and whenever $i, j \in \mathbf{N}$ and $\alpha \in \mathbf{N}^{N-2}$ satisfy $|\alpha|<i,|\alpha|<j$, then

$$
\Phi_{5}\left(e_{\alpha} \otimes e_{i} \otimes e_{j}\right)=\sum_{|\beta|=|\alpha|} \mu_{\alpha, \beta} e_{\beta} \otimes e_{i} \otimes e_{j} .
$$

Proof. Disjointifying for $\ell_{p_{1}} \hat{\otimes} \cdots \hat{\otimes} \ell_{p_{N-1}}$ as in Step 2, we can assume without loss of generality that whenever $i, j \in \mathbf{N}, \alpha \in \mathbf{N}^{N-2}$ satisfy $|\alpha|<i<j$, then

$$
\Phi\left(e_{\alpha} \otimes e_{i} \otimes e_{j}\right)=\sum_{|\beta|<i} \lambda_{\alpha, \beta, i} e_{\beta} \otimes e_{i} \otimes e_{j} .
$$

Apply Basic Lemma 2 to the sequence $\left\{\lambda_{\alpha, \beta, i}\right\}$ and assume that (after factoring $\Phi$ through $K_{\sigma} \Phi J_{\sigma}$ and renaming it $\Phi$ again) this sequence satisfies the conclusions of that lemma.

Let $L_{\alpha}=\left[e_{\alpha} \otimes e_{i} \otimes e_{j}: i, j \in \mathbf{N}\right]$ with projection $P_{\alpha}$. Since $\ell_{p_{1}} \hat{\otimes} \cdots \hat{\otimes} \ell_{p_{N-2}}$ has a basis consisting of $e_{\alpha}$ 's, then $\left(L_{\alpha}\right)$ forms a Schauder decomposition for $\ell_{p_{1}} \hat{\otimes} \cdots \hat{\otimes} \ell_{p_{N}}$.

Define $\tilde{\Phi} \in B\left(\ell_{p_{1}} \hat{\otimes} \cdots \hat{\otimes} \ell_{p_{N}}\right)$ as follows:

$$
P_{\beta} \tilde{\Phi}\left(e_{\alpha} \otimes e_{i} \otimes e_{j}\right)= \begin{cases}\lambda_{\alpha, \beta} e_{\beta} \otimes e_{i} \otimes e_{j} & \text { if }|\alpha| \vee|\beta|<i<j \\ P_{\beta} \Phi\left(e_{\alpha} \otimes e_{i} \otimes e_{j}\right) & \text { otherwise }\end{cases}
$$


Let $\alpha, \beta \in \mathbf{N}^{N-2} ; \epsilon_{\alpha, \beta}=\sum_{i>|\alpha| \vee|\beta|} \epsilon_{\alpha, \beta, i}$ and $x \in L_{\alpha} ;$ i.e.,

$$
x=\sum_{i} \sum_{j} c_{i, j} e_{\alpha} \otimes e_{i} \otimes e_{j} .
$$

Then,

$$
\begin{aligned}
P_{\beta} \Phi x-P_{\beta} \tilde{\Phi} x & \\
= & \sum_{\imath>|\alpha| \vee|\beta|}\left(\sum_{j=i+1}^{\infty} c_{i, j}\left[P_{\beta} \Phi\left(e_{\alpha} \otimes e_{i} \otimes e_{j}\right)-P_{\beta} \tilde{\Phi}\left(e_{\alpha} \otimes e_{i} \otimes e_{j}\right)\right]\right) \\
& =\sum_{i>|\alpha| \vee|\beta|}\left(\lambda_{\alpha, \beta, i}-\lambda_{\alpha, \beta}\right) e_{\beta} \otimes e_{i} \otimes\left(\sum_{j=i+1}^{\infty} c_{i, j} e_{j}\right) .
\end{aligned}
$$

Since $\left\|\sum_{j=i+1}^{\infty} c_{i, j} e_{j}\right\| \leq\|x\|$

$$
\left\|P_{\beta} \Phi x-P_{\beta} \tilde{\Phi} x\right\| \leq \epsilon_{\alpha, \beta}\|x\| \text {. }
$$

If we choose $\epsilon_{\alpha, \beta}$ small enough so that $\sum_{\alpha, \beta} \epsilon_{\alpha, \beta}<\frac{\epsilon}{2}$, then $\tilde{\Phi}$ is well defined and satisfies $\|\Phi-\tilde{\Phi}\|<\frac{\epsilon}{2}$; moreover, whenever $|\alpha|<i<j$, we have

$$
\tilde{\Phi}\left(e_{\alpha} \otimes e_{i} \otimes e_{j}\right)=\sum_{|\beta|<i} \lambda_{\alpha, \beta} e_{\beta} \otimes e_{i} \otimes e_{j}
$$

Let $T: L_{\alpha} \rightarrow L_{\beta}$ be defined by $T(x)=P_{\beta} \tilde{\Phi} x . T$ is clearly a bounded map and $L_{\alpha} \equiv L_{\beta} \equiv \ell_{p_{N-1}} \hat{\otimes} \ell_{p_{N}}$. It follows that if $|\alpha| \vee|\beta|<i<j$ then $T\left(e_{\alpha} \otimes e_{i} \otimes e_{j}\right)=\lambda_{\alpha, \beta} e_{\beta} \otimes e_{i} \otimes e_{j}$. Since all the arguments work if the maximum is attained at the $(N-1)$-st coordinate and the next maximum is attained in the last coordinate, we can also assume that if $|\alpha| \vee|\beta|<j<i$ then $T\left(e_{\alpha} \otimes e_{i} \otimes e_{j}\right)=\mu_{\alpha, \beta} e_{\beta} \otimes e_{i} \otimes e_{j}$. Thus $T$ takes value $\lambda_{\alpha, \beta}$ in the upper triangular part of a copy of $\ell_{p_{N-1}} \hat{\otimes} \ell_{p_{N}}$ and the value $\mu_{\alpha, \beta}$ in the lower part. Since we assumed that

$$
\frac{1}{p_{N-1}}+\frac{1}{p_{N}} \leq 1
$$

we have that $\lambda_{\alpha, \beta}=\mu_{\alpha, \beta}$. (If $\lambda_{\alpha, \beta} \neq \mu_{\alpha, \beta}$, then $\left(T-\mu_{\alpha, \beta} I\right) /\left(\lambda_{\alpha, \beta}-\mu_{\alpha, \beta}\right)$ would be a projection onto the upper triangular part of $\ell_{p_{N-1}} \hat{\otimes} \ell_{p_{N}}$, contradicting. Theorem 1.3.)

Let $\alpha=\left(\alpha_{1}, \cdots, \alpha_{N-2}\right)$ and $\gamma=\left(\alpha_{N-1}, \alpha_{N}\right)$. We now have that if $\alpha<\gamma$, then

$$
\tilde{\Phi}\left(e_{\alpha} \otimes e_{\gamma}\right)=\sum_{\beta<\gamma} \lambda_{\alpha, \beta} e_{\beta} \otimes e_{\gamma} .
$$


Define a map $\Psi \in B\left(\ell_{p_{1}} \hat{\otimes} \cdots \hat{\otimes} \ell_{p_{N-2}}\right)$, as in Step 4 , by

$$
\left(\Psi e_{\alpha}, e_{\beta}\right)=\lambda_{\alpha, \beta}
$$

Since $\Psi$ is bounded, we apply Basic Lemma 1 to it and assume without loss of generality (after factoring $K_{\sigma} \tilde{\Phi} J_{\sigma}$ and then renaming it $\tilde{\Phi}$ again) that if $x \in \partial M_{n}$ (here $\partial M_{n}$ is a subset of $\ell_{p_{1}} \hat{\otimes} \cdots \hat{\otimes} \ell_{p_{N-2}}$ ) and $m \neq n$,

$$
\left\|Q_{m} \Psi x\right\| \leq \epsilon_{n, m}\|x\|
$$

Let $L_{\alpha}=\left[e_{\alpha} \otimes e_{i} \otimes e_{j}: i, j \in \mathbf{N}\right]$ with projection $P_{\alpha}$, and define $\Phi_{1} \in$ $B\left(\ell_{p_{1}} \hat{\otimes} \cdots \hat{\otimes} \ell_{p_{N}}\right)$ as follows

$$
\Phi_{5}\left(e_{\alpha} \otimes e_{\gamma}\right)= \begin{cases}\left(Q_{|\alpha|} \Psi e_{\alpha}\right) \otimes e_{\gamma} & \text { if } \alpha<\gamma \\ \tilde{\Phi}\left(e_{\alpha} \otimes e_{\gamma}\right) & \text { otherwise. }\end{cases}
$$

Let $x \in L_{\alpha},|\alpha|=n$; i.e., $x=\sum_{\gamma} c_{\gamma} e_{\alpha} \otimes e_{\gamma}$. Hence,

$$
\begin{aligned}
\tilde{\Phi} x-\Phi_{5} x & =\sum_{\gamma>\alpha}\left[c_{\gamma} \tilde{\Phi} e_{\alpha} \otimes e_{\gamma}-c_{\gamma}\left(Q_{n} \Psi e_{\alpha}\right) \otimes e_{\gamma}\right] \\
& =\sum_{\substack{k=1 \\
k \neq n}}^{\infty}\left(Q_{k} \Psi e_{\alpha}\right) \otimes\left(\sum_{\gamma>n, k} c_{\gamma} e_{\alpha} \otimes e_{\gamma}\right) .
\end{aligned}
$$

Since $\left\|\sum_{\gamma>n, k} c_{\gamma} e_{\alpha} \otimes e_{\gamma}\right\| \leq\|x\|$, the result follows.

The induction step is an extension of Step 5.

Step 6. Let $\Phi \in B\left(\ell_{p_{1}} \hat{\otimes} \cdots \hat{\otimes} \ell_{p_{N}}\right)$ be such that whenever $\alpha \in \mathbf{N}^{k}, \gamma \in \mathbf{N}^{N-k}$ satisfy $\alpha<\gamma$, then $\Phi\left(e_{\alpha} \otimes e_{\gamma}\right)=\sum_{|\beta|=|\alpha|} \lambda_{\alpha, \beta} e_{\beta} \otimes e_{\gamma}$. Then for every $\epsilon>0$, there exist $\sigma=\left(\sigma_{1}, \sigma_{2}, \cdots, \sigma_{N}\right)$ and $\Phi_{k} \in B\left(\ell_{p_{1}} \hat{\otimes} \cdots \hat{\otimes} \ell_{p_{N}}\right)$ such that $\left\|\Phi_{k}-K_{\sigma} \Phi J_{\sigma}\right\|<\epsilon$ and whenever $\alpha \in \mathbf{N}^{k-1}$ and $\gamma \in \mathbf{N}^{N-k+1}$ satisfy $\alpha<\gamma$, we have

$$
\Phi_{k}\left(e_{\alpha} \otimes e_{\gamma}\right)=\sum_{|\beta|=|\alpha|} \mu_{\alpha, \beta} e_{\beta} \otimes e_{\gamma}
$$

Sketch of proof. Disjointifying as in Step 2 we assume that whenever $\alpha \in$ $\mathbf{N}^{k-1}, i \in \mathbf{N}$ and $\gamma \in \mathbf{N}^{N-k}$ satisfy $\alpha<i<\gamma$, then

$$
\Phi\left(e_{\alpha} \otimes e_{i} \otimes e_{\gamma}\right)=\sum_{\beta<i} \lambda_{\alpha, \beta, i} e_{\beta} \otimes e_{i} \otimes e_{\gamma} .
$$

Assume also that the sequence $\left\{\lambda_{\alpha, \beta, i}\right\}$ satisfies the conclusion of Basic Lemma 2. 
Let $\alpha \in \mathbf{N}^{k-1} ; L_{\alpha}=\left[e_{\alpha} \otimes e_{i} \otimes e_{\gamma}: i \in \mathbf{N}, \gamma \in \mathbf{N}^{N-k}\right]$ with projection $P_{\alpha}$ and define $\tilde{\Phi}$ as in Step 5. Since for every $i_{0},\left\|\sum_{\gamma>i_{0}} c_{i_{0}, \gamma} e_{i_{0}} \otimes e_{\gamma}\right\| \leq$ $\left\|\sum_{i, \gamma} c_{i, \gamma} e_{i} \otimes e_{\gamma}\right\|$, then $\|\Phi-\tilde{\Phi}\|<\epsilon$ and whenever $\alpha \in \mathbf{N}^{k-1}, \gamma \in \mathbf{N}^{N-k}$ satisfy $\alpha<i<\gamma$, then

$$
\tilde{\Phi}\left(e_{\alpha} \otimes e_{i} \otimes e_{\gamma}\right)=\sum_{\beta<i} \lambda_{\alpha, \beta} e_{\alpha} \otimes e_{i} \otimes e_{\gamma} .
$$

Fix $\alpha, \beta \in \mathbf{N}^{k-1}$ and define $T: L_{\alpha} \rightarrow L_{\beta}$ by $T x=P_{\beta} \tilde{\Phi} x$. Since $T$ is bounded and $L_{\alpha} \equiv L_{\beta} \equiv \ell_{p_{k}} \hat{\otimes} \cdots \hat{\otimes} \ell_{p_{N}}$ we assume that $T$ is defined on $Z=\ell_{p_{k}} \hat{\otimes} \cdots \hat{\otimes} \ell_{p_{N}}$.

Decompose $Z$ into $\left(E_{j}\right)_{j=k}^{N}$, where $E_{j}=\left[e_{\theta}: \theta_{j}<\theta_{i}\right.$ for every $\left.i \neq j\right]$; (i.e., $E_{j}$ is the span of those $e_{\theta}$ where the minimum occurs at the $j$ th coordinate). For instance, if $e_{\theta} \in E_{k}$, then $e_{\theta}=e_{i} \otimes e_{\gamma}$ for some $i<\gamma$ and hence $T e_{\theta}=$ $\lambda_{\alpha, \beta} e_{\theta}=\lambda^{(k)} e_{\theta}$. Since all the arguments work for the other permutations of the coordinates we can assume that there exist $\lambda^{(j)}$ such that if $x \in E_{j}$, then $T x=\lambda^{(j)} x, j=k, k+1, \cdots, N$.

We will use that $\frac{1}{p_{i}}+\frac{1}{p_{j}} \leq 1$ for every $i \neq j$ to conclude that the $\lambda^{(j)}$ 's have to be equal. Indeed, let $\bar{m}=(m, m, \cdots, m) \in \mathbf{N}^{N-k-3}$ and consider $K_{m}=\left[e_{i} \otimes e_{j} \otimes e_{\bar{m}}: i, j \leq m\right]$. It is clear that $K_{m} \equiv \ell_{p_{k}}^{m} \hat{\otimes} \ell_{p_{k+1}}^{m}$ and that $T$ restricted to it gives us $\lambda^{(k)}$ in the upper triangular part and $\lambda^{(k+1)}$ in the lower one. If $\lambda^{(k)} \neq \lambda^{(k+1)}$, we would have that the $m$-triangular parts are uniformly complemented and this is not true. A similar argument proves that the $\lambda^{(j)}$ 's are all equal.

In conclusion, if $\alpha \in \mathbf{N}^{k-1}, \gamma \in \mathbf{N}^{N-k+1}$, and $\alpha<\gamma$, then

$$
\tilde{\Phi}\left(e_{\alpha} \otimes e_{\gamma}\right)=\sum_{\beta<\gamma} \lambda_{\alpha, \beta} e_{\beta} \otimes e_{\gamma} .
$$

Define $\Psi \in B\left(\ell_{p_{1}} \hat{\otimes} \cdots \hat{\otimes} \ell_{p_{k-1}}\right)$ by $\left(\Psi e_{\alpha}, e_{\beta}\right)=\lambda_{\alpha, \beta}$; apply Basic Lemma 1 to it and finish the proof as in Step 5.

Iterating Step 6 we finish the proof of the proposition.

We will see in the next section that, for most cases, if $\frac{1}{p_{\imath}}+\frac{1}{p_{j}}>1, X$ is not primary. This is not always true, however.

Theorem 3.3. Let $1 \leq p<\infty$ and $n \in \mathbf{N}$. Then $X=\ell_{p} \hat{\otimes} \cdots \hat{\otimes} \ell_{p}$ (n times) is primary.

Proof. We divide the proof into two cases. If $\frac{2}{p} \leq 1$, this is a particular case of Theorem 3.1. If $\frac{2}{p}>1$ then the triangular projections are bounded. This implies that the "tetrahedrals" are complemented. (An example of this is $\mathcal{T}=\left[e_{\alpha}: \alpha_{1}<\alpha_{2}<\cdots<\alpha_{n}\right]$.) Since all of them are isometrically isomorphic and there are finitely many of them we conclude that 
$X \approx\left[e_{\alpha}: \alpha_{1}<\alpha_{2}<\cdots<\alpha_{n}\right]$ by Pełczyński's decomposition method. Then the proofs of Theorem 3.1 and Proposition 3.2 apply to this space.

The proof of Theorem 3.1 dualizes (formally) to $X_{*}=\ell_{q_{1}} \check{\otimes} \cdots \check{\otimes} \ell_{q_{N}}$. Define $J_{\sigma}$ and $K_{\sigma}$ on $B\left(\ell_{q_{1}} \check{\otimes} \cdots \check{\otimes} \ell_{q_{N}}\right)$ as in $B\left(\ell_{p_{1}} \hat{\otimes} \cdots \dot{\otimes} \ell_{p_{N}}\right)$. The key to the dualization argument is that $\left(J_{\sigma}\right)^{*}=K_{\sigma}$ and $\left(K_{\sigma}\right)^{*}=J_{\sigma}$.

Theorem 3.4. Let $X_{*}=\ell_{q_{1}} \check{\otimes} \cdots \check{\otimes} \ell_{q_{N}}$ be such that $\frac{1}{q_{i}}+\frac{1}{q_{j}} \geq 1$ for every $i \neq j$. Then $X_{*}$ is primary.

Proof. Let $\Phi \in B\left(\ell_{q_{1}} \check{\otimes} \cdots \check{\otimes} \ell_{q_{N}}\right)$, and $0<\epsilon<\frac{1}{2}$. Then $\Phi^{*} \in B\left(\ell_{p_{1}} \hat{\otimes} \cdots \dot{\otimes} \ell_{p_{N}}\right)$ and whenever $i \neq j$ we have $\frac{1}{p_{i}}+\frac{1}{p_{j}} \leq 1$. Therefore, Theorem 3.1 tells us that there exist $\sigma$ and $\lambda \in \mathbf{C}$ such that $\left\|K_{\sigma} \Phi^{*} J_{\sigma}-\lambda I_{X}\right\|<\epsilon$.

Since $K_{\sigma} \Phi^{*} J_{\sigma}-\lambda I_{X}=\left(K_{\sigma} \Phi J_{\sigma}-\lambda I_{X_{*}}\right)^{*}$ we have that $\left\|K_{\sigma} \Phi J_{\sigma}-\lambda I_{X_{*}}\right\|<\epsilon$. Therefore, $\Phi$ or $I_{X_{*}}-\Phi$ factors through $X_{*}$, and since $X_{*}$ is isomorphic to its $r^{\prime}$-sum, we conclude that $X_{*}$ is primary.

\section{4. $\ell_{p}$ subspaces of $\ell_{p_{1}} \hat{\otimes} \cdots \hat{\otimes} \ell_{p_{N}}$.}

Theorem 1.3 tells us that $\ell_{p}$ embeds into $\ell_{p_{1}} \hat{\otimes} \cdots \hat{\otimes} \ell_{p_{N}}$ if there exists a nonempty $A \subset\{1, \cdots, N\}$ for which $p=r_{A}$, where $\frac{1}{r_{A}}=\min \left\{1, \sum_{i \in A} \frac{1}{p_{i}}\right\}$. We will see in the next theorem that the converse holds.

Theorem 4.1. $\quad \ell_{p}$ embeds into $\ell_{p_{1}} \hat{\otimes} \cdots \hat{\otimes} \ell_{p_{N}}$ if and only if there exists a non-empty $A \subset\{1, \cdots, N\}$ such that $p=r_{A}$.

We will use this theorem to prove the following:

Theorem 4.2. Let $X=\ell_{p_{1}} \hat{\otimes} \cdots \hat{\otimes} \ell_{p_{N}}$ and assume that for some $i \neq j$, $\frac{1}{p_{i}}+\frac{1}{p_{j}}>1$ and that $p_{k} \notin\left\{r_{A}: k \notin A\right\}$ for $k=i, j$. Then $X$ is not primary.

Remark. Theorem 4.1 could probably be generalized to characterize when $m$-fold tensor products embed into $n$-fold tensor products for $m \leq n$ and this would slightly improve Theorem 4.2:

We will use Theorem 1.3 to decompose $X \approx\left[e_{\alpha}: \alpha_{i}>\alpha_{j}\right] \oplus\left[e_{\alpha}: \alpha_{i} \leq \alpha_{j}\right]$. The condition $p_{i} \notin\left\{r_{A}: i \notin A\right\}$ insures that $\ell_{p_{i}}$ does not embed into $\left[e_{\alpha}: \alpha_{i} \leq \alpha_{j}\right.$ ]. (This is easily seen for example when $N=2$. In this case $X=\ell_{p_{1}} \hat{\otimes} \ell_{p_{2}}$ and $p_{k} \notin\left\{r_{A}: k \notin A\right\}$ means $p_{1} \neq p_{2}$; we can then observe that $\ell_{p_{1}}$ does not embed into $\left[e_{i} \otimes e_{j}: i \leq j\right]$.)

We will prove Theorem 4.1 by induction. Assume for the remainder of this section that $X=\ell_{p_{1}} \hat{\otimes} \cdots \hat{\otimes} \ell_{p_{N}} ; \frac{1}{r}=\min \left\{1, \sum_{i=1}^{N} \frac{1}{p_{i}}\right\}$ and that $\Phi: \ell_{p} \rightarrow$ 
$\ell_{p_{1}} \hat{\otimes} \cdots \hat{\otimes} \ell_{p_{N}}$ is an isomorphism. We can assume without loss of generality that there is a sequence of increasing natural numbers $n_{i}$ such that

$$
\Phi e_{i} \in\left[e_{\alpha}: n_{i}<|\alpha|<n_{i+1}\right] \quad \text { for every } i \text {. }
$$

If $p>1$ this is true because $\Phi e_{j} \rightarrow 0$ weakly. If $p=1$ and $P_{M_{n}}$ is the projection onto $\left[e_{\alpha}: \alpha \leq n\right]$, we can find infinitely many pairs of $e_{i}$ 's (say $e_{k}$ and $\left.e_{l}\right)$ such that $P_{M_{n}} \Phi\left(e_{k}-e_{l}\right) \approx 0$. Then we replace the $e_{i}$ 's by differences of unit vectors and get $(*)$.

We say that $\Psi: \ell_{p} \rightarrow \ell_{p}$ is an $\ell_{p}$-average isometry if there exist a sequence of subsets of $\mathbf{N}, \sigma_{1}<\sigma_{2}<\cdots$ and scalars $a_{k}$ such that

$$
\Psi e_{i}=\sum_{k \in \sigma_{i}} a_{k} e_{k} \quad \text { and } \quad \sum_{k \in \sigma_{i}}\left|a_{k}\right|^{p}=1 \quad \text { for every } i .
$$

Finally we will let $E_{n}=\left[e_{\alpha}: \min \{\alpha\} \leq n\right]$ for every $n \in \mathbf{N}$. The key to the induction step is that

$$
E_{n} \approx\left(\ell_{p_{2}} \hat{\otimes} \cdots \hat{\otimes} \ell_{p_{N}}\right) \oplus\left(\ell_{p_{1}} \hat{\otimes} \ell_{p_{3}} \hat{\otimes} \cdots \hat{\otimes} \ell_{p_{N}}\right) \oplus \cdots \oplus\left(\ell_{p_{1}} \hat{\otimes} \cdots \hat{\otimes} \ell_{p_{N-1}}\right) .
$$

(The isomorphism constant goes to infinity with $n$.) Notice that each one of those summands is an $(N-1)$-projective tensor product.

We need two lemmas.

Lemma 4.3. Let $\Phi: \ell_{p} \rightarrow \ell_{p_{1}} \hat{\otimes} \cdots \hat{\otimes} \ell_{p_{N}}$ be as in $(*)$ with $p>r$. Then for every $\epsilon>0$ we can find $n \in \mathbf{N}$ such that $\left\|\left(I-P_{E_{n}}\right) \Phi\right\| \leq \epsilon$.

Lemma 4.4. Let $\Phi: \ell_{p} \rightarrow \ell_{p_{1}} \hat{\otimes} \cdots \hat{\otimes} \ell_{p_{N}}$ be as in (*) with $p<r$, then for every $\epsilon>0$ there exists $\Psi: \ell_{p} \rightarrow \ell_{p}$ an $\ell_{p}$-average isometry such that $\|\Phi \Psi\|<\epsilon$.

Proof of Theorem 4.1. The theorem is clearly true for $N=1$. Assume that the result is true for $(N-1)$-projective tensor products and let $\Phi: \ell_{p} \rightarrow$ $\ell_{p_{1}} \hat{\otimes} \cdots \hat{\otimes} \ell_{p_{N}}$ be an isomorphism satisfying $(*)$.

It follows from Lemma 4.4 that $p \geq r$. If $p=r$ there is nothing to prove since $\ell_{p}$ clearly embeds in the main diagonal. If $p>r$, Lemma 4.3 tells us that $\Phi \ell_{p}$ is essentially inside $E_{n}$ and therefore it is inside one of the $(N-1)$ tensor products. Hence it has to be of the form $r_{A}$ for some nonempty $A$ by induction.

We used in the proof the well-known fact that if $\ell_{p}$ embeds into $X \oplus Y$ then $\ell_{p}$ embeds into $X$ or into $Y$.

For the proof of Theorem 4.2 we need one more lemma. 
Lemma 4.5. Let $X=\ell_{p_{1}} \hat{\otimes} \cdots \hat{\otimes} \ell_{p_{N}}, i, j \leq N, i \neq j$ and assume that $p_{i} \notin\left\{r_{A}: i \notin A\right\}$. Then $\ell_{p_{i}}$ does not embed into $\left[e_{\alpha}: \alpha_{i} \leq \alpha_{j}\right]$.

Proof of Theorem 4.2. Use Theorem 1.3 to decompose $X \approx\left[e_{\alpha}: \alpha_{i}>\alpha_{j}\right] \oplus$ $\left[e_{\alpha}: \alpha_{i} \leq \alpha_{j}\right]$. Lemma 4.5 tells us that $\ell_{p_{j}}$ does not embed into $\left[e_{\alpha}: \alpha_{i}>\alpha_{j}\right]$, and that $\ell_{p_{i}}$ does not embed into $\left[e_{\alpha}: \alpha_{i} \leq \alpha_{j}\right]$. Therefore neither of them is isomorphic to $X$, and so $X$ is not primary.

Proof of Lemma 4.3. If the lemma were false, we could find some $\epsilon_{0}>0$; a sequence of normalized vectors $\left\{x_{i}\right\}_{i \in \mathbf{N}}$ in $\ell_{p}$ satisfying $\operatorname{supp}\left\{x_{i}\right\}<\operatorname{supp}\left\{x_{i+1}\right\}$ for every $i$; and an increasing sequence $n_{i} \in \mathbf{N}$ satisfying $\left\|P_{i} \Phi x_{i}\right\| \geq \epsilon_{0}$ where $P_{i}$ is the projection onto the diagonal block

$$
\left[e_{\alpha}: n_{i} \leq \alpha<n_{i+1}\right] \text {. }
$$

Theorem 3.1 implies that $\left[P_{i} \Phi x_{i}: i \in \mathbf{N}\right] \approx \ell_{r}$. Let $P$ be the diagonal projection onto $\left[P_{i} \Phi x_{i}: i \in \mathbf{N}\right]$ and consider $P \Phi: \ell_{p} \rightarrow \ell_{r}$. Since $\left\|P \Phi e_{i}\right\| \geq \epsilon_{0}$ for every $i \in \mathbf{N}$ we have that $P \Phi$ is not compact. This is a contradiction.

Sketch of the proof of Lemma 4.4. For $N=1$ the result is easy. The condition (*) says that $\Phi: \ell_{p} \rightarrow \ell_{r}$ is diagonal; i.e., $\Phi e_{i}=\lambda_{i} e_{i}$. Moreover since $\Phi$ is bounded, there exists $M>0$ such that $\left|\lambda_{i}\right| \leq M$ for every $i$. We get the blocks by taking the $a_{k}$ 's constant in every $\sigma$. Let $\sigma \subset \mathbf{N}$ be of cardinality $n$ (say). Then $\left\|\sum_{k \in \sigma}\left(\frac{1}{n}\right)^{1 / p} e_{k}\right\|_{p}=1$ but $\left\|\sum_{k \in \sigma}\left(\frac{1}{n}\right)^{1 / p} \Phi e_{k}\right\|_{r} \leq M n^{1 / r-1 / p}$ goes to zero as $n$ goes to infinity.

Assume the result for $N-1$ and let $\Phi: \ell_{p} \rightarrow \ell_{p_{1}} \hat{\otimes} \cdots \hat{\otimes} \ell_{p_{N}}$ be as in (*). The idea is to find an $\ell_{p}$-average isometry $\Psi \in B\left(\ell_{p}\right)$ such that $\Phi \Psi$ is essentially supported in a diagonal block; then since the diagonal block is like $\ell_{r}$, the case $N=1$ takes care of it.

To find $\Psi$ we have to find an increasing sequence $n_{i} \in \mathbf{N}$ and a normalized sequence $\left\{x_{i}\right\}_{i \in \mathbf{N}}$ in $\ell_{p}$ satisfying $\operatorname{supp}\left\{x_{i}\right\} \leq \operatorname{supp}\left\{x_{i+1}\right\}$ for every $i \in \mathbf{N}$ and $\Phi x_{i} \in\left[e_{\alpha}: n_{i} \leq \alpha<n_{i+1}\right]$. (The last inclusion is an "almost" inclusion; that is, for a given $\epsilon_{i}>0$ there exists $n_{i} \in \mathbf{N}$ such that the distance from $\Phi x_{i}$ to $\left[e_{\alpha}: n_{i} \leq \alpha<n_{i+1}\right]$ is less that $\epsilon_{i}$.)

It is clear that it is enough to do this for $x_{1}$ and $x_{2}$ because we can iterate it to conclude the lemma. Clearly $\Phi x_{1} \in\left[e_{\alpha}: \alpha<n\right]$ for some $n$. We want to find $x_{2}$ such that $\Phi x_{2}$ is supported outside $E_{n}$. Since $E_{n}$ is isomorphic to the sum of $(N-1)$-projective tensor products, we can apply the induction step to insure the existence of $x_{2}$.

Sketch of the proof of Lemma 4.5. The proof of this goes by induction too. The result is clear for $N=2$. Suppose it is true for $N-1$ and false for $N$. 
Then let $Z=\left[e_{\alpha}: \alpha_{i} \leq \alpha_{j}\right] \subset \ell_{p_{1}} \hat{\otimes} \cdots \hat{\otimes} \ell_{p_{N}}$ and, by the assumption, find $\Phi: \ell_{p_{i}} \rightarrow Z$, an isomorphism satisfying $(*)$.

The main diagonal of $Z$ is isomorphic to $\ell_{r}$ and $p_{i}>r$. Hence, by Lemma 4.3 , there exists $n \in \mathbf{N}$ such that $\Phi \ell_{p_{i}}$ is essentially inside $E_{n}$. We will look at the $N$-summands of $Z \cap E_{n}$ to get a contradiction.

One of those summands does not contain the $i$ th component and hence is isomorphic to

$$
\ell_{p_{1}} \hat{\otimes} \cdots \hat{\otimes} \ell_{p_{i-1}} \hat{\otimes} \ell_{p_{i+1}} \hat{\otimes} \cdots \hat{\otimes} \ell_{p_{N}} .
$$

The condition $p_{i} \notin\left\{r_{A}: i \notin A\right\}$ and Theorem 4.1 imply that $\ell_{p_{i}}$ does not embed there.

Another summand does not contain the $j$ th component. This really means that $\alpha_{j} \leq n$. Therefore, $\alpha_{i} \leq n$ as well and the summand is isomorphic to $\hat{\otimes}_{k \neq i, j} \ell_{p_{k}}$. We conclude as before that $\ell_{p_{i}}$ does embed here.

The remaining summands will have the same structure but with $N-1$ terms. Then the induction hypothesis implies that $\ell_{p_{i}}$ does not embed into any one of them.

Therefore, $\ell_{p_{i}}$ does not embed in $Z$. This is a contradiction.

\section{Primarity of Polynomials and Operator Spaces.}

In this section we discuss the primarity of $\left(\ell_{p_{1}} \hat{\otimes} \cdots \hat{\otimes} \ell_{p_{N}}\right)^{*}$. There will be really only one case to consider; namely that of $r=1$ (recall that $\frac{1}{r}=$ $\left.\min \left\{1, \sum_{i=1}^{N} \frac{1}{p_{i}}\right\}\right)$, which we demonstrate below using techniques of Bourgain [Bo] and Blower [B].

It is interesting to note that completely different factors determine the primarity of $\left(\ell_{p_{1}} \hat{\otimes} \cdots \hat{\otimes} \ell_{p_{N}}\right)^{*}$ when $r=1$ and $r>1$. When $r>1$ it is the unboundedness of the main triangle projection in each pair (taken separately) that is the most important factor, while for $r=1$ we will see that the main point is that we have $\ell_{\infty}$-blocks down the diagonal.

Theorem 5.1. Let $X=\ell_{p_{1}} \hat{\otimes} \cdots \hat{\otimes} \ell_{p_{N}}$ be such that $\frac{1}{r}=\min \left\{1, \sum_{i=1}^{N} \frac{1}{p_{i}}\right\}=$ 1. Then $\left(\ell_{p_{1}} \hat{\otimes} \cdots \hat{\otimes} \ell_{p_{N}}\right)^{*}$ is primary.

This result will solve the question of primarity for spaces of polynomials. Since the space of analytic polynomials of degree $m$ on $\ell_{p}$ is isomorphic (with constant $\frac{m^{m}}{m !}$ ) to the dual of the symmetric $m$-fold tensor product $\hat{\otimes}_{s}^{m} \ell_{p}$. That is $\mathcal{P}_{m} \approx\left(\hat{\otimes}_{s}^{m} \ell_{p}\right)^{*}$. (Here $m$ is the number of times that one takes the tensor product.)

Lemma 5.2. For any $1 \leq p<\infty$ and $m \in \mathbf{N}$ we have that $\ell_{p} \hat{\otimes} \cdots \hat{\otimes} \ell_{p} \approx$ 
$\hat{\otimes}_{s}^{m} \ell_{p}$.

Proof. We use Pełczyński's decomposition method again. Since $\ell_{p} \hat{\otimes} \cdots \hat{\otimes} \ell_{p}$ is isomorphic to its infinite $s$-sum $\left(s=\max \left\{1, \frac{p}{m}\right\}\right)$ we only have to prove that they embed complementably into each other. It is clear that $\hat{\otimes}_{s}^{m} \ell_{p}$ embeds into $\ell_{p} \hat{\otimes} \cdots \hat{\otimes} \ell_{p}$. Indeed, $S \in B\left(\ell_{p} \hat{\otimes} \cdots \hat{\otimes} \ell_{p}\right)$ defined by $S e_{\alpha}=$ $\frac{1}{m !} \sum_{\pi \in \Pi_{m}} e_{\pi(\alpha)}$ shows that the embedding is 1-complemented. On the other hand, for $i \leq N$ let $\sigma_{i}(j)=m(j-1)+i, \sigma=\left(\sigma_{1}, \cdots, \sigma_{m}\right)$ and define $T \in B\left(\ell_{p} \hat{\otimes} \cdots \hat{\otimes} \ell_{p}\right)$ by $T=K_{\sigma} S J_{\sigma}$. It is clear that $T$ factors through $\hat{\otimes}_{s}^{m} \ell_{p}$ and it is easy to see that $T e_{\alpha}=\frac{1}{m !} e_{\alpha}$. Hence, $\ell_{p} \hat{\otimes} \cdots \hat{\otimes} \ell_{p}$ embeds complementably into $\hat{\otimes}_{s}^{m} \ell_{p}$ and the result follows.

Corollary 5.3. Let $1 \leq p<\infty$ and $m \geq 1$. The space of homogeneous analytic polynomials $\mathcal{P}_{m}\left(\ell_{p}\right)$ and the symmetric tensor product of $m$ copies of $\ell_{p}$ are primary.

We now proceed to the proof of the theorem. Notice that if

$$
X=\ell_{p_{1}} \hat{\otimes} \cdots \hat{\otimes} \ell_{p_{N}}
$$

is such that $\frac{1}{r}=\min \left\{1, \sum_{i=1}^{N} \frac{1}{p_{i}}\right\}=1$, then Theorem 2.2 tells us that

$$
\left(\ell_{p_{1}} \hat{\otimes} \cdots \hat{\otimes} \ell_{p_{N}}\right)^{*} \approx\left(\sum_{n=1}^{\infty} \ell_{q_{1}}^{n} \check{\otimes} \cdots \check{\otimes} \ell_{q_{N}}^{n}\right)_{\infty} .
$$

This decomposition allows us to use the technique developed by Bourgain [Bo] to prove that $H^{\infty}$ is primary; namely, one obtains the general theorem from the finite dimensional version.

The proof is an exact generalization of the proof of Blower [B] that $B(H)$ is primary; it has no surprises, and so we will simply sketch the part that is different for the case $N>2$, and refer the interested reader to [B] for other details. The proof follows from the following 2 lemmas, as indicated in [Bo].

Proposition 5.4. Given $n \in \mathbf{N}, \epsilon>0$ and $K<\infty$, there exists $N_{0}=$ $N_{0}(n, \epsilon, K)$ such that if $M \geq N_{0}$ and $T \in B\left(\ell_{q_{1}}^{M} \check{\otimes} \cdots \check{\otimes} \ell_{q_{N}}^{M}\right)$ with $\|T\| \leq K$, then there exist subsets $\sigma_{1}, \sigma_{2}, \cdots \sigma_{N} \subset\{1, \cdots, M\}$ of cardinality $n$, and a constant $\lambda$ such that if $\sigma=\left(\sigma_{1}, \sigma_{2}, \cdots, \sigma_{N}\right)$ then,

$$
\left\|K_{\sigma} T J_{\sigma}-\lambda I_{n}\right\| \leq \epsilon .
$$

Thus, one of $K_{\sigma} T J_{\sigma}$ and $K_{\sigma}\left(I_{N}-T\right) J_{\sigma}$ is invertible.

Remark. Here $J_{\sigma}: \ell_{q_{1}}^{n} \check{\otimes} \cdots \check{\otimes} \ell_{q_{N}}^{n} \rightarrow \ell_{q_{1}}^{M} \check{\otimes} \cdots \check{\otimes} \ell_{q_{N}}^{M}$ is defined by $J_{\sigma} e_{\alpha}=$ $e_{\sigma(\alpha)}$ where $\sigma(\alpha)=\left(\sigma_{1}\left(\alpha_{1}\right), \cdots, \sigma_{N}\left(\alpha_{N}\right)\right)$, and $\sigma_{i}=\left\{\sigma_{i}(1), \sigma_{i}(2), \cdots, \sigma_{i}(n)\right\}$. Moreover, $\sigma_{i}(k)<\sigma_{i}(l)$ iff $k<l$. The definition for $K_{\sigma}$ is similar. 
Proposition 5.5. Given $n \in \mathbf{N}$ and $\epsilon>0$ there exists $N_{0}=N_{0}(n, \epsilon)$ such that if $M \geq N_{0}$ and $E$ is an $n$-dimensional subspace of $\ell_{q_{1}}^{M} \check{\otimes} \cdots \check{\otimes} \ell_{q_{N}}^{M}$ then there exists a subspace $F$ of $\ell_{q_{1}}^{M} \ddot{\otimes} \cdots \check{\otimes} \ell_{q_{N}}^{M}$, isometrically isomorphic to $\ell_{q_{1}}^{n} \check{\otimes} \cdots \check{\otimes} \ell_{q_{N}}^{n}$, and a block projection $Q$ from $\ell_{q_{1}}^{M} \check{\otimes} \cdots \check{\otimes} \ell_{q_{N}}^{M}$ to $F$ such that $\|Q x\|<\epsilon\|x\|$ for every $x \in E$.

Sketch of the proof of Proposition 5.4. Let $T \in B\left(\ell_{q_{1}}^{M} \check{\otimes} \cdots \check{\otimes} \ell_{q_{N}}^{M}\right)$ such that $\|T\| \leq K$. We will find a copy of $\ell_{q_{1}}^{n} \check{\otimes} \cdots \check{\otimes} \ell_{q_{N}}^{n}$ inside $\ell_{q_{1}}^{M} \check{\otimes} \cdots \check{\otimes} \ell_{q_{N}}^{M}$ such that $T$ is essentially a multiple of the identity when restricted to this subspace. We accomplish this in two steps.

Step 1. Find a large subset $\psi \subset\{1, \cdots, M\}$ and $\lambda \in \mathbf{C}$ such that whenever $\alpha=\left(\alpha_{1}, \cdots, \alpha_{N}\right)$ is such that $\alpha_{1}<\cdots<\alpha_{N}$ and $\alpha_{k} \in \psi$ for $i \leq N$, then $\left|\left(T e_{\alpha}, e_{\alpha}\right)-\lambda\right|<\epsilon$.

Step 2. Find $\sigma_{1}<\sigma_{2}<\cdots<\sigma_{N} \subset \psi$ each of cardinality $n$, such that whenever $\alpha=\left(\alpha_{1}, \cdots, \alpha_{N}\right), \alpha^{\prime}=\left(\alpha_{1}^{\prime}, \cdots, \alpha_{N}^{\prime}\right)$ are are such that $\alpha_{k}, \alpha_{k}^{\prime} \in \sigma_{k}$ for every $k \leq N$ and $\alpha \neq \alpha^{\prime}$, then $\left|\left(T e_{\alpha}, e_{\alpha^{\prime}}\right)\right|<\epsilon$. Then define $\mathcal{S}=$ $\left[e_{\alpha}: \alpha_{k} \in \sigma_{k}\right]$. One can easily verify that if $\epsilon>0$ is chosen small enough then $T$ restricted and projected into $\mathcal{S}$ is essentially a multiple of the identity and that $\mathcal{S}$ is isometrically isomorphic to $\ell_{q_{1}}^{n} \check{\otimes} \cdots \check{\otimes} \ell_{q_{N}}^{n}$.

Both steps depend on Ramsey's Theorem and they are very minor modifications of Blower's argument.

For Step 1 divide the disk $\{z:|z| \leq K\}$ into finitely many disjoint subsets $V_{k}$ of diameter less than $\epsilon$, and define the coloring on $N$-sets of $\{1, \cdots, M\}$ by $\left\{\alpha_{1}, \cdots, \alpha_{N}\right\} \rightarrow \ell$ if $\left(T e_{\alpha}, e_{\alpha}\right) \in V_{\ell}$ where $\alpha=\left(\alpha_{1}, \cdots, \alpha_{N}\right)$ for $\alpha_{1}<$ $\cdots<\alpha_{N}$. Then use Ramsey's Theorem fo find a large monochromatic set $\psi$.

The proof of Step 2 involves many different cases (but all of them are similar). One has to look at all the different ways that $\left(\alpha_{1}, \cdots, \alpha_{N}\right) \neq$ $\left(\alpha_{1}^{\prime}, \cdots, \alpha_{N}^{\prime}\right)$. We will illustrate the case when $\alpha_{k}<\alpha_{k}^{\prime}$ for every $k \leq N$.

Color the $2 N$-elements of $\{1, \cdots, M\}$ by: $\left\{\alpha_{1}, \alpha_{1}^{\prime}, \alpha_{2}, \alpha_{2}^{\prime}, \cdots, \alpha_{N}, \alpha_{N}^{\prime}\right\}$ is bad if $\alpha_{1}<\alpha_{1}^{\prime}<\alpha_{2}<\alpha_{2}^{\prime}<\cdots<\alpha_{N}<\alpha_{N}^{\prime}$ and $\left|\left(T e_{\alpha}, e_{\alpha^{\prime}}\right)\right| \geq \epsilon$ where $\alpha=\left(\alpha_{1}, \cdots, \alpha_{N}\right)$ and $\alpha^{\prime}=\left(\alpha_{1}^{\prime}, \cdots, \alpha_{N}^{\prime}\right)$; it is good otherwise.

Ramsey's Theorem gives us a large monochromatic subset $\psi_{1} \subset \psi$. We will show that $\psi_{1}$ has to be good. Let $\alpha_{1}^{\prime}<\alpha_{2}<\alpha_{2}^{\prime}<\cdots<\alpha_{N}<\alpha_{N}^{\prime}$ be the $2 N-1$ largest elements of $\psi_{1}$, and let $\beta=\left(\alpha_{2}, \cdots, \alpha_{N}\right), \alpha^{\prime}=\left(\alpha_{1}^{\prime}, \cdots, \alpha_{N}^{\prime}\right)$, and $F=\left[e_{i} \otimes e_{\beta}: i \in \psi_{1}, i<\alpha_{1}^{\prime}\right]$.

It is clear that $F \equiv \ell_{p_{1}}^{\left|\psi_{1}\right|-2 N+1}$. Define $\tilde{T}: F \rightarrow \mathbf{C}$ by $\tilde{T}(x)=\left(T x, e_{\alpha^{\prime}}{ }^{\prime}\right)$ Then we have that $\tilde{T}$ is a map from $\ell_{p_{1}}^{s}$ into $\mathbf{C}$, with norm less than or equal to $K$ and maps the canonical basis into "large" elements. Since we assumed that $p_{1}>1$ this is a contradiction.

Now we have to look at all the other possibilities; e.g., $\alpha_{1}^{\prime}>\alpha_{1}$ and 
$\alpha_{k}<\alpha_{k}^{\prime}$ for $2 \leq k \leq N$ etc. We have to look also at the cases when some of the coordinates are equal, but these are not very different. We prove the proposition by choosing $M$ large enough.

Sketch of the proof of Proposition 5.5. It is enough to prove that if $x \in$ $\ell_{q_{1}}^{M} \check{\otimes} \cdots \check{\otimes} \ell_{q_{N}}^{M}$ with $\|x\| \leq 1$, then we can find $Q$, a large block projection, such that $\|Q(x)\| \leq \epsilon$. Then take an $\epsilon$-net of the sphere of $E,\left\{x_{i}\right\}_{i=1}^{s}$. Find $Q_{1}$ a large block projection such that $\left\|Q_{1} x_{1}\right\| \leq \epsilon$; then find $Q_{2}$ a large block projection contained in the range of $Q_{1}$ such that $\left\|Q_{2} Q_{1} x_{2}\right\| \leq \epsilon$. Proceeding in this way we get that $Q=Q_{s} \cdots Q_{2} Q_{1}$; this $Q$ does it.

To check the first claim let $x \in \ell_{q_{1}}^{M} \check{\otimes} \cdots \check{\otimes} \ell_{q_{N}}^{M}$ with $\|x\| \leq 1$ and let $\rho>0$ (to be fixed later). Then define a coloring on the $N$ sets of $\{1, \cdots, M\}$ by: $\left\{\alpha_{1}, \cdots, \alpha_{N}\right\}$ is bad if $\left|\left(x, e_{\alpha}\right)\right| \geq \rho$ where $\alpha=\left(\alpha_{1}, \cdots, \alpha_{N}\right)$ and $\alpha_{1}<\cdots<$ $\alpha_{N}$. And good otherwise. Ramsey's theorem gives us a large monochromatic subset, and this subset has to be good.

\section{Appendix}

In this section we will prove Basic Lemmas 1 and 2 from Section 3.

Proof of Basic Lemma 1. For this proof let $M_{n}=\left[e_{\alpha}: \alpha \leq n\right]$ with projection $P_{n}$. We will divide the proof into two parts, one for $m>n$ and the other one for $m<n$. In both cases, $\sigma=\left(\sigma_{1}, \cdots, \sigma_{N}\right)$ satisfies $\sigma_{1}=\sigma_{2}=\cdots=\sigma_{N}$.

The case $m>n$ is simpler; we start with it.

If $K \subset \ell_{p_{1}} \hat{\otimes} \cdots \hat{\otimes} \ell_{p_{N}}$ is a compact set, then $K$ is essentially inside one of the $M_{n}$ 's. The following elementary lemma states this fact quantitatively (we omit its proof as it is an easy exercise). The proof of the case $m>n$ follows easily from it.

Lemma 6.1. Let $K \subset \ell_{p_{1}} \hat{\otimes} \cdots \hat{\otimes} \ell_{p_{N}}$ be a compact set and $\epsilon_{k}>0$ be given. Then we can find a sequence $n_{k} \in \mathbf{N}$ such that $\sup _{x \in K}\left\|\left(I-P_{n_{k}}\right) x\right\|<\epsilon_{k}$.

We start the inductive construction of $\sigma_{1}$. Set $A_{1}=\mathbf{N}$ and $\sigma_{1}(1)=$ $\min A_{1}$. Let $K=\Phi$ Ball $\partial M_{\sigma_{1}(1)}$ and $\epsilon_{k}=\epsilon_{1, k}$. Then find $A_{2} \subset A_{1} \backslash\left\{\sigma_{1}(1)\right\}$ according to Lemma 6.1 ; and set $\sigma_{1}(2)=\min A_{2}$.

Let $K=\Phi$ Ball $\partial M_{\sigma_{1}(2)}, \epsilon_{k}=\epsilon_{2, k}$ and find $A_{3} \subset A_{2} \backslash\left\{\sigma_{1}(2)\right\}$ according to Lemma 6.1. Then set $\sigma_{1}(3)=\min A_{3}$.

Continuing in this fashion we get $\sigma_{1}$ and construct $\sigma=\left(\sigma_{1}, \cdots, \sigma_{1}\right)$. It is easy to see that if $x \in \partial M_{n}$ and $m>n$, then

$$
\left\|Q_{m} K_{\sigma} \Phi J_{\sigma} x\right\| \leq \epsilon_{n, m}\|x\|
$$


We will now prove the case $m<n$.

The construction of $\sigma_{1}$ is similar to the previous case. We need the following elementary lemma (which as before do not prove).

Lemma 6.2. Let $1<p<\infty, F$ a finite dimensional space, and $T: \ell_{p} \rightarrow F$ a bounded linear map. Then for every $\epsilon>0$, the set $\left\{i:\left\|T e_{i}\right\|>\epsilon\right\}$ is finite.

We will only present the induction step for the construction of $\sigma_{1}$. Assume that $\Lambda \subset \mathbf{N}$ is an infinite set with first $n$ elements $\sigma_{1}(1), \cdots, \sigma_{1}(n)$. We want to find an infinite $\Lambda^{\prime} \subset \Lambda$ with the same first $n$ elements as $\Lambda$ such that whenever $\alpha \in\left(\Lambda^{\prime}\right)^{N}$ is such that $e_{\alpha} \notin M_{\sigma_{1}(n)}$, then $\left\|P_{\sigma_{1}(n)} \Phi e_{\alpha}\right\|<\epsilon$. Then we will choose $\sigma_{1}(n+1)=\min \Lambda^{\prime} \backslash\left\{\sigma_{1}(1), \cdots, \sigma_{1}(n)\right\}$.

The construction of $\Lambda^{\prime}$ uses Ramsey's Theorem as in Section 5. We look at all the different ways that $e_{\alpha} \notin M_{\sigma_{1}(n)}$. We will illustrate this for two different cases. The others are very similar.

Case 1. $\sigma_{1}(n)<\alpha_{1}<\alpha_{2}<\cdots<\alpha_{N}$.

Color the $N$-sets of $\left\{i \in \Lambda: i>\sigma_{1}(n)\right\}$ as follows: $\left\{\alpha_{1}, \cdots, \alpha_{N}\right\}$ is good if $\alpha_{1}<\cdots<\alpha_{N}$ and $\left\|P_{\sigma_{1}(n)} \Phi e_{\alpha}\right\|<\epsilon$ and bad otherwise.

Ramsey's Theorem gives us a monochromatic infinite set $\Lambda_{1} \subset \Lambda$. It is easy to see that Lemma 6.2 implies that the set has to be good. (Let $\beta_{1}<$ $\cdots<\beta_{N-1}$ be the $N-1$ smallest elements of $\Lambda_{1}$ and define $T: \ell_{p_{N}} \rightarrow M_{\sigma_{1}(n)}$ as follows: if $i>\beta_{N-1}$, then $T e_{i}=P_{\sigma_{1}(n)} \Phi e_{\left(\beta_{1}, \cdots, \beta_{N-1}, i\right)}$ and if $i \leq \beta_{N-1}$, then $T e_{i}=0$. If $\Lambda_{1}$ were $b a d$ this would contradict Lemma 6.2.)

Case 2. $\alpha_{1}, \alpha_{2} \leq \sigma_{1}(n)<\alpha_{3}<\cdots<\alpha_{N}$.

Color the $(N-2)$-sets of $\left\{i \in \Lambda: i>\sigma_{1}(n)\right\}$ as follows: $\left\{\alpha_{3}, \cdots, \alpha_{N}\right\}$ is good if $\alpha_{3}<\cdots<\alpha_{N}$ and $\left\|P_{\sigma_{1}(n)} \Phi e_{\alpha}\right\|<\epsilon$ for every $\alpha_{1}, \alpha_{2} \leq \sigma_{1}(n)$, (notice that $\left.\alpha=\left(\alpha_{1}, \alpha_{2}, \alpha_{3}, \cdots, \alpha_{N}\right)\right)$ and bad otherwise.

Once again Ramsey's Theorem gives an infinite monochromatic subset of $\Lambda$. And as before it has no choice but to be good. This follows because there are only finitely many $\alpha_{1}, \alpha_{2} \leq \sigma_{1}(n)$.

There are finitely many ways in which $e_{\alpha} \notin M_{\sigma_{1}(n)}$. They are very similar to the two cases just considered, and repeating the above argument for all of them we get $\tilde{\Lambda} \subset \Lambda$ that is good in all the cases. Then let $\Lambda^{\prime}=\tilde{\Lambda} \bigcup\left\{\sigma_{1}(1), \cdots, \sigma_{1}(n)\right\}$. We choose $\epsilon>0$ small enough so that whenever $x \in \partial M_{n+1}$, then

$$
\left\|P_{\sigma_{1}(n)} K_{\sigma} \Phi J_{\sigma} x\right\| \leq \min _{k \leq n}\left\{\epsilon_{n+1, k}\right\}\|x\| .
$$

Proof of Basic Lemma 2. Assume that we have a sequence of complex numbers $\left\{\lambda_{\alpha, \beta, j}: \alpha, \beta \in \mathbf{N}^{N-1},|\alpha| \vee|\beta|<j\right\}$ and a sequence of positive numbers, 
$\left\{\epsilon_{\alpha, \beta, j}: j>|\alpha| \vee|\beta|\right\}$

For $\alpha, \beta$ fixed, find a subsequence $\left\{j_{k}\right\}$ of $\{j: j>|\alpha| \vee|\beta|\}$ and some $\lambda_{\alpha, \beta} \in \mathbf{C}$ satisfying:

$$
\begin{gathered}
\lim _{j_{k} \rightarrow \infty} \lambda_{\alpha, \beta, j_{k}}=\lambda_{\alpha, \beta} \\
\left|\lambda_{\alpha, \beta, j_{k}}-\lambda_{\alpha, \beta}\right|<\epsilon_{\alpha, \beta, k} .
\end{gathered}
$$

Moreover, if we have finitely many $\left\{\alpha_{l}, \beta_{l}\right\}_{l \leq m}$, we can find a subsequence $\left\{j_{k}\right\}$ such that $(* *)$ is true for every $l \leq m$.

The condition $j>|\alpha| \vee|\beta|$ is the key to extend the argument to all $\alpha, \beta \in \mathbf{N}^{N-1}$. The basic idea is that once we have fixed $\sigma_{1}(1), \cdots, \sigma_{1}(n)$, we take the subsequence $j_{k}$ from $\left\{j: j>\sigma_{1}(n)\right\}$; hence, we do not affect the initial segment.

We will only present the induction step for $\sigma_{1}$. Assume that $\Lambda \subset \mathbf{N}$ is an infinite set with first elements $\sigma_{1}(1), \sigma_{1}(2), \cdots, \sigma_{1}(n)$. We want to find an infinite $\Lambda^{\prime} \subset \Lambda$ with the first $n$ elements as in $\Lambda$, and such that (**) is satisfied for every $\alpha, \beta \leq \sigma_{1}(n)$. We can do that because there are only finitely many of them. We take the subsequence $j_{k}$ from $\left\{j \in \Lambda: j>\sigma_{1}(n)\right\}$ and let $\Lambda^{\prime}=\left\{j_{k}: k \in \mathbf{N}\right\} \bigcup\left\{\sigma_{1}(1), \cdots, \sigma_{1}(n)\right\}$. Then set $\sigma_{1}(n+1)=j_{1}$, the minimum of the $j_{k}$ 's (remember that $j_{1}>\sigma_{1}(n)$ ).

Repeating the process we finish the proof.

\section{References}

[AEO] D. Alspach, P. Enflo and E. Odell, On the structure of $\mathcal{L}_{p},(1<p<\infty)$, Studia Math., 60 (1977), 79-90.

[Ar1] J. Arazy, Basic sequences, embeddings, and the uniqueness of the symmetric structure in unitary spaces, J. Func. Anal., 40 (1981), 302-340.

[Ar2] —_ On subspaces of $c_{p}$ which contain $c_{p}$, Compositio Math., 41 (1980), 297336.

[A] A. Arias, Nest algebras in the trace class, J. of Operator Theory., 29 (1993), 389-409.

[Be] G. Bennett, Unconditional convergence and almost everywhere convergence, $\mathrm{Z}$. Wah. verw. Gebiete, 34 (1976) 135-155.

[B] G. Blower, The space $B(H)$ is primary, Bull. London Math. Soc., 22 (1990), 176-182.

[Bo] J. Bourgain, On the primarity of $\mathcal{H}^{\infty}$-spaces, Israel J. Math., 45 (1983), 329-336.

[CL] P. Casazza and B. Lin, Projections on Banach spaces with symmetric bases, Studia Math., 52 (1974), 189-193.

[FJ] J. Farmer and W.B. Johnson, Polynomial Schur and polynomial Dunford-Pettis properties, Banach Spaces, Contemporary Mathematics, 144 (1993), Bor-Luh Lin, (ed.), Amer. Math. Soc., Providence, RI. 
[JRZ] W.B. Johnson, H.P. Rosenthal, and M. Zippin, On bases, finite dimensional decompositions, and weaker structures in Banach spaces, Israel J. Math., 9 (1971), 488-506.

[KP] S. Kwapien and A. Pełczyński, The main triangular projection in matrix spaces and its applications, Studia Math., 34 (1970), 43-68.

[LP] J. Lindenstrauss and A. Pełczyński, Absolutely summing operators in $\mathcal{L}_{p}$-spaces and their applications, Studia Math., 29 (1968) 275-326.

[LT] J. Lindenstrauss and L. Tzafriri, Classical Banach spaces I: Sequence spaces, SpringerVerlag, Berlin, (1977).

[MN] B. Maurey and A. Nahoum: Note aux C.R. Acad. Sc. Paris, t. 276, March 1973, page 751 .

[M] J. Mujica, Complex analysis in Banach spaces, Notas de Mathematica, vol. 120, North-Holland, Amsterdam, 1986.

[R] R. Ryan, Applications of topological tensor products to infinite dimensional holomorphy, Ph.D. Thesis, Trinity College, Dublin, 1980.

[Z] I. Zalduendo, An estimate for multilinear forms on $\ell_{p}$ spaces, preprint.

Received August 10, 1993. This research was partially supported by NSF DMS-8921369. Portions of this paper form a part of the second author's Ph.D. dissertation under the supervision of W.B. Johnson.

UNIVERSITY OF TEXAS

SAN ANTONio, TX 78249

E-mail address: arias@ringer.cs.utsa.edu

AND

UNIVERSity OF NorThern Colorado

Greeley, CO 80639 



\title{
PACIFIC JOURNAL OF MATHEMATICS
}

\author{
Founded in 1951 by
}

\author{
E. F. Beckenbach (1906-1982) $\quad$ F. Wolf (1904-1989)
}

\section{EDITORS}

Sun-Yung A. Chang (Managing Editor) Robert Finn University of California

Los Angeles, CA 90095-1555

pacific@math.ucla.edu

\section{F. Michael Christ}

University of California

Los Angeles, CA 90095-1555

christ@math.ucla.edu

Nicholas Ercolani

University of Arizona

Tucson, AZ 85721

ercolani@math.arizona.edu
Stanford University

Stanford, CA 94305

finn@gauss.stanford.edu

Steven Kerckhoff

Stanford University

Stanford, CA 94305

spk@gauss.stanford.edu

Martin Scharlemann

University of California

Santa Barbara, CA 93106

mgscharl@math.ucsb.edu

\section{Gang Tian}

Massachusettes Institute of Technology

Cambridge, MA 02139

tian@math.mit.edu

\section{V.S. Varadarajan}

University of California

Los Angeles, CA 90095-1555

vsv@math.ucla.edu

Dan Voiculescu

University of California

Berkeley, CA 94720

dvv@math.berkeley.edu

\section{SUPPORTING INSTITUTIONS}

CALIF. INST. OF TECHNOLOGY CHINESE UNIV. OF HONG KONG MACQUARIE UNIVERSITY NEW MEXICO STATE UNIV. OREGON STATE UNIV. PEKING UNIVERSITY RITSUMEIKAN UNIVERSITY STANFORD UNIVERSITY TOKYO INSTITUTE OF TECHNOLOGY UNIVERSIDAD DE LOS ANDES
UNIV. OF ARIZONA

UNIV. OF BRITISH COLUMBIA

UNIV. OF CALIF., BERKELEY

UNIV. OF CALIF., DAVIS

UNIV. OF CALIF,, IRVINE UNIV. OF CALIF., LOS ANGELES

UNIV. OF CALIF., RIVERSIDE UNIV. OF CALIF., SAN DIEGO UNIV. OF CALIF., SANTA BARBARA UNIV. OF CALIF., SANTA CRUZ
UNIV. OF HAWAII

UNIV. OF MELBOURNE

UNIV. OF MONTANA

UNIV. NACIONAL AUTONOMA DE MEXICO

UNIV. OF NEVADA, RENO

UNIV. OF OREGON

UNIV. OF SOUTHERN CALIFORNIA

UNIV OF UTAH

UNIV. OF WASHINGTON

WASHINGTON STATE UNIVERSITY

The supporting Institutions listed above contribute to the cost of publication of this Journal, but they are not owners or publishers and have no responsibility for its contents or policies.

Manuscripts must be prepared in accordance with the instructions provided on the inside back cover.

The table of contents and the abstracts of the papers in the current issue, as well as other information about the Pacific Journal of Mathematics, may be found on the Internet at http://www.math.uci.edu/pjm.html.

The Pacific Journal of Mathematics (ISSN 0030-8730) is published monthly except for July and August. Regular subscription rate: $\$ 245.00$ a year (10 issues). Special rate: $\$ 123.00$ a year to individual members of supporting institutions.

Subscriptions, back issues published within the last three years and changes of subscribers address should be sent to Pacific Journal of Mathematics, P.O. Box 4163, Berkeley, CA 94704-0163, U.S.A. Prior back issues are obtainable from Kraus Periodicals Co., Route 100, Millwood, NY 10546.

The Pacific Journal of Mathematics at the University of California, c/o Department of Mathematics, 981 Evans Hall, Berkeley, CA 94720 (ISSN 0030-8730) is published monthly except for July and August. Second-class postage paid at Berkeley, CA 94704, and additional mailing offices. POSTMASTER: send address changes to Pacific Journal of Mathematics, P.O. Box 6143, Berkeley, CA 94704-0163.

PUBLISHED BY PACIFIC JOURNAL OF MATHEMATICS at University of California, Berkeley, CA 94720, A NON-PROFIT CORPORATION

This publication was typeset using AMS-LATEX,

the American Mathematical Society's TEX macro system.

Copyright (c) 1995 by Pacific Journal of Mathematics 


\title{
PACIFIC JOURNAL OF MATHEMATICS
}

\author{
Volume $175 \quad$ No. $1 \quad$ September 1996
}

Homogeneous Ricci positive 5-manifolds

Dimitri Alekseevsky, Isabel Dotti de Miatello and CARlos J.

FERRARIS

On the structure of tensor products of $\ell_{p}$-spaces

ALVARO ARIAS and JEFFREY D. FARMER

The closed geodesic problem for compact Riemannian 2-orbifolds

JosEPH E. BORZELLINO and BENJAMIN G. LORICA

Small eigenvalue variation and real rank zero

Ola Bratteli and George A. Elliott

Global analytic hypoellipticity of $\square_{b}$ on circular domains

So-CHIN CHEN

Sharing values and a problem due to C. C. Yang

XIN-Hou HuA

Commutators and invariant domains for Schrödinger propagators

Min-JEI HUANG

Chaos of continuum-wise expansive homeomorphisms and dynamical properties of sensitive maps of graphs

\section{HiS AO KATO}

Some properties of Fano manifolds that are zeros of sections in homogeneous vector bundles over Grassmannians

\section{OLIVER KÜCHLE}

On polynomials orthogonal with respect to Sobolev inner product on the unit circle

XIN Li and FRANCISCO MARCELLAN

Maximal subfields of $\mathbf{Q}(i)$-division rings

STEVEN LIEDAHL

Virtual diagonals and $n$-amenability for Banach algebras

AlAN L. T. PATERSON

Rational Pontryagin classes, local representations, and $K^{G}$-theory

Claude Schochet

An equivalence relation for codimension one foliations of 3-manifolds

SANDRA SHIELDS

A construction of Lomonosov functions and applications to the invariant subspace problem

\section{ALEKSANDER SIMONIČ}

Complete intersection subvarieties of general hypersurfaces 